\title{
Energy shift of the three-particle system in a finite volume
}

\author{
Jin-Yi Pang, ${ }^{1}$ Jia-Jun Wu, ${ }^{2,1}$ Hans-Werner Hammer, ${ }^{3}$ Ulf-G. Meißner, ${ }^{1,4,5}$ and Akaki Rusetsky ${ }^{1}$ \\ ${ }^{1}$ HISKP and BCTP, Rheinische Friedrich-Wilhelms Universität Bonn, 53115 Bonn, Germany \\ ${ }^{2}$ School of Physical Sciences, University of Chinese Academy of Sciences, Beijing 100049, China \\ ${ }^{3}$ Institut für Kernphysik, Technische Universität Darmstadt, 64289 Darmstadt, Germany and ExtreMe \\ Matter Institute EMMI, GSI Helmholtzzentrum für Schwerionenforschung, 64291 Darmstadt, Germany \\ ${ }^{4}$ Institute for Advanced Simulation (IAS-4), Institut für Kernphysik (IKP-3) and Jülich Center for Hadron \\ Physics, Forschungszentrum Jülich, D-52425 Jülich, Germany \\ ${ }^{5}$ Ivane Javakhishvili Tbilisi State University, 0186 Tbilisi, Georgia
}

(Received 6 February 2019; published 29 April 2019)

\begin{abstract}
Using the three-particle quantization condition recently obtained in the particle-dimer framework, the finite-volume energy shift of the two lowest three-particle scattering states is derived up to and including order $L^{-6}$. Furthermore, assuming that a stable dimer exists in the infinite volume, the shift for the lowest particle-dimer scattering state is obtained up to and including order $L^{-3}$. The result for the lowest threeparticle state agrees with the results from the literature, and the result for the lowest particle-dimer state reproduces the one obtained by using the Lüscher equation.
\end{abstract}

DOI: 10.1103/PhysRevD.99.074513

\section{INTRODUCTION}

Recent years have seen a rapid progress in developing the formalism for the analysis of the lattice data in the threeparticle sector [1-28] (for a recent review, see Ref. [29]). One of the main goals of this development was the derivation of the so-called quantization condition, i.e., the equation that relates the finite-volume energy spectrum with the infinite-volume observables [1,5,6,18,21]. In particular, in our previous papers $[17,18,20]$ we have proposed an approach to the problem that is based on the use of the nonrelativistic effective theory and the particle-dimer picture in a finite volume (a very similar approach was proposed in Refs. [14,21]). Within this approach, a tower of particle-dimer couplings with increasing inverse mass dimension, describing the three-particle force, is fit to the lattice spectrum in the three-particle sector. The physical observables are then constructed through solving the scattering equations in the infinite volume, using the input determined on the lattice. It has been shown [18] that this approach is conceptually equivalent to other approaches in the literature.

In case of two particles, the infinite-volume spectrum consists of a continuum and (possibly) bound states below the two-particle threshold. In a finite volume, this all

Published by the American Physical Society under the terms of the Creative Commons Attribution 4.0 International license. Further distribution of this work must maintain attribution to the author(s) and the published article's title, journal citation, and DOI. Funded by SCOAP. translates into a discrete spectrum. If there were no interactions, the momenta of the particles in a box of size $L$ would be quantized, according to $\mathbf{p}_{i}=2 \pi \mathbf{n}_{i} / L, \mathbf{n}_{i} \in \mathbb{Z}^{3}$ for $i=1,2$, and the spectrum would be given by a sum of the free energies of two particles. In the presence of interactions, the energy levels are displaced from their free values. In this case, the spectrum consists of the levels that in the infinite-volume limit end up in the continuum (we refer to these levels as to the scattering states), and the levels that are continuously transformed into the bound states in this limit. This displacement of the scattering states can be calculated in a systematic expansion in $1 / L$, by using the two-particle quantization condition (Lüscher equation) [30]. The final result for the lowest scattering state takes the form [31-34]

$$
\begin{aligned}
\Delta E_{2}= & E_{2}(L)-2 m \\
= & \frac{4 \pi a}{m L^{3}}\left\{1+c_{1}\left(\frac{a}{\pi L}\right)+c_{2}\left(\frac{a}{\pi L}\right)^{2}+c_{3}\left(\frac{a}{\pi L}\right)^{3}\right. \\
& \left.+\frac{2 \pi r a^{2}}{L^{3}}-\frac{\pi a}{m^{2} L^{3}}\right\}+O\left(L^{-7}\right),
\end{aligned}
$$

where, for simplicity, we display the formula for the displacement of the ground-state energy levels of two identical particles with the mass $m$. In this formula, $a$ and $r$ denote the two-body $\mathrm{S}$-wave scattering length and the effective range, respectively, and $c_{1}, c_{2}, c_{3}$ are known numerical constants. The last term is the relativistic correction, which vanishes, as $m \rightarrow \infty$. The mixing to other partial waves does not enter at this order in $1 / L$. 
The spectrum of the three-particle system is richer. If the two-body interaction is attractive and sufficiently strong, bound state(s) [or stable dimer(s)] may be formed and, apart of the three-particle scattering states, we shall have particle-dimer scattering states as well. ${ }^{1}$ In particular, as shown in Ref. [20], the finite-volume energy levels in such a system display characteristic avoided level crossings at those values of $L$, where the free energies of the threeparticle and particle-dimer systems coincide.

As in the two-particle case, the energy levels of an interacting three-body system in a finite volume are shifted from their free values. The expression for the energy shift of the lowest three-particle scattering state is known in the literature up to and including $O\left(L^{-6}\right)$ (see, e.g., Refs. [8,9,31-34]) and even up to and including $O\left(L^{-7}\right)$, see Ref. [35]. The formula up to and including $O\left(L^{-6}\right)$ takes the form

$$
\begin{aligned}
\Delta E_{3}= & E_{3}(L)-3 m \\
= & \frac{12 \pi a}{m L^{3}}\left\{1+d_{1}\left(\frac{a}{\pi L}\right)+d_{2}\left(\frac{a}{\pi L}\right)^{2}+\frac{3 \pi a}{m^{2} L^{3}}+\frac{6 \pi r a^{2}}{L^{3}}\right. \\
& \left.+\left(\frac{a}{\pi L}\right)^{3} d_{3} \ln \frac{m L}{2 \pi}\right\}-\frac{D}{48 m^{3} L^{6}}+O\left(L^{-7}\right),
\end{aligned}
$$

where $d_{1}, d_{2}, d_{3}$ are the known numerical constants, the quantity $D$ contains the contribution of the three-body force and the fourth term inside the brackets, containing inverse power of $m$, is a relativistic correction. The expression for the excited three-particle levels, as well as the particledimer levels (in case these exist), to the best of our knowledge, are not available in the literature. Our present work fills this gap. In particular, we derive the finite volume shift of the lowest three-particle and particle-dimer scattering states in a systematic expansion in powers of $1 / L$ (including logarithms), using the approach of Ref. [18]. Note that we do not consider three-particle bound states (trimers) which were discussed in Refs. $[2,10,17]$ and thus in the following refer to the lowest scattering state as "ground state" for convenience.

The justification of our enterprise is twofold. First and foremost, we provide closed analytic expressions for the energy shift of different levels in a finite volume, which can be easily fitted to the lattice data. Moreover, these expressions are written directly in terms of infinite-volume

\footnotetext{
${ }^{1}$ Following the terminology widely used in the literature, throughout this paper we use the name "dimer" in two different contexts. Namely, in this paragraph, a "stable dimer" is merely equivalent to the notion of the two-body bound state (a stable particle). In a more general context, a "dimer" is an auxiliary field with given quantum numbers that enables one to transform the three-particle equations to a simpler form. The existence of a stable particle is no more required (of course, if such a particle exists, the two interpretations are equivalent). We expect that the terminology used here does not lead to confusion.
}

observables (like two-body effective-range expansion parameters of the three-body threshold amplitude). On the contrary, fitting the numerical solution of the quantization condition directly to the lattice data is much more complicated and less transparent. The second argument in favor of our study is that deriving the perturbative shifts of different levels is a powerful check of the approach, since, e.g., the result for the ground state has been already available in the literature.

In short, our result for the three-particle scattering states has the following form:

$$
\begin{aligned}
E_{3}(L)-E_{3}^{\text {free }}(L)= & \frac{P_{0}}{L^{3}}+\frac{P_{1}}{L^{4}}+\frac{P_{2}}{L^{5}}+\frac{P_{3}}{L^{6}} \ln \frac{m L}{2 \pi} \\
& +\frac{P_{4}}{L^{6}}+O\left(L^{-7}\right),
\end{aligned}
$$

where $E_{3}^{\text {free }}(L)$ is the energy of three free particles in a box (the ground state or the first excited state in the center-ofmass frame), whereas the coefficients $P_{0}, \ldots, P_{4}$ are different for different levels and different irreps of the octahedral group. As mentioned before, these coefficients depend only on the infinite-volume physical observables. Moreover, we have found the formula of the ground-state shift of a particle and a tightly bound stable dimer:

$$
E_{1 d}(L)-E_{1 d}^{\mathrm{free}}(L)=\frac{\mathcal{A}_{d}}{L^{3}}+O\left(L^{-4}\right),
$$

where $\mathcal{A}_{d}$ denotes the particle-dimer scattering amplitude at threshold.

An explicit result for the energy shift of the three-body ground state is given in Eq. (39), while the results for first excited state are given in Eq. (78) for the $A_{1}^{+}$representation and Eq. (105) for the $E^{+}$representation. Compact, easy to use formulas with numerical coefficients for these energy shifts are given in the Appendix A. Finally, the shift for the lowest particle-dimer level is given in Eq. (50). In the course of our work, we also obtain a simple and transparent closed expression for the three-particle contribution of the three-body scattering amplitude at threshold. The lattice practitioners, who are only interested in the final results, are directly referred to the Appendix A.

The plan of our paper is rather straightforward. First, in Sec. II we write down a basis matrix identity that enables one to derive the perturbative expansion of the energy shift in powers of $1 / L$. Next, in Sec. III we derive the formula for the shift of the three-particle ground state up to and including $L^{-6}$. In Sec. IV we address the calculation of the particle-dimer levels to leading order. In Sec. V, we calculate the first excited three-particle energy level shift up to and including $L^{-6}$. Section VI is dedicated to the comparison to previous results in the literature. Finally, Sec. VII contains our conclusions and an outlook. All technical details are relegated to the Appendixes. 


\section{TRANSFORMING THE QUANTIZATION CONDITION}

The quantization condition for three identical spinless bosons, given in Refs. [18,20], is obtained under certain assumptions, namely: (i) the particles obey the nonrelativistic dispersion law and (ii) only S-wave interactions are taken into account. The work to go beyond these assumptions is already in progress, but for the time being we restrict ourselves to the existing framework.

Under these assumptions, the quantization condition takes the form $\operatorname{det} \mathcal{A}=0$, where the matrix $\mathcal{A}$ is defined in momentum space:

$$
\mathcal{A}_{p q}=8 \pi \tau_{L}^{-1}(\mathbf{p} ; E) L^{3} \delta_{\mathbf{p q}}-8 \pi Z(\mathbf{p}, \mathbf{q} ; E) \doteq\left(\tau^{-1}-Z\right)_{p q} .
$$

Here, the three-momenta $\mathbf{p}, \mathbf{q}$ take the discrete values, $\mathbf{p}=2 \pi \mathbf{n} / L, \mathbf{n}=\mathbb{Z}^{3}$ (similarly for $\mathbf{q}$ ). A cutoff $\Lambda$ is imposed, demanding that the matrix is finite, with $\mathbf{p}$, $\mathbf{q}<\Lambda$. Note also that writing down the quantization condition in the matrix notation, we do not show the factors $L^{3}$ explicitly. An overall factor $8 \pi$ is included in the definition of $\tau_{p q}^{-1}$ and $Z_{p q}$. The sum over each momentum index $p$ (not boldface) always implies the factor $1 / L^{3}$, whereas $\delta_{p q}=L^{3} \delta_{\mathbf{p q}}$. Further,

$$
\begin{aligned}
8 \pi \tau_{L}^{-1}(\mathbf{p} ; E) & =p^{*} \cot \delta\left(p^{*}\right)+S\left(\mathbf{p},\left(p^{*}\right)^{2}\right), \\
S\left(\mathbf{p},\left(p^{*}\right)^{2}\right) & =-\frac{4 \pi}{L^{3}} \sum_{\mathbf{l}} \frac{1}{\mathbf{p}^{2}+\mathbf{p l}+\mathbf{l}^{2}-m E},
\end{aligned}
$$

and $p^{*}$ is the magnitude of the relative momentum of the pair in the rest frame, with

$$
\left(p^{*}\right)^{2}=m E-\frac{3}{4} \mathbf{p}^{2}
$$

In Eq. (6) the momentum sum is implicitly regularized by using dimensional regularization, leading eventually to the pertinent Lüscher zeta-function. ${ }^{2}$ Further, $\delta\left(p^{*}\right)$ is the $\mathrm{S}$-wave phase shift in the two-particle subsystem. The effective range expansion reads

$$
p^{*} \cot \delta\left(p^{*}\right)=-\frac{1}{a}+\frac{1}{2} r\left(p^{*}\right)^{2}+O\left(\left(p^{*}\right)^{4}\right),
$$

where $a, r$ are the two-body scattering length and the effective range, respectively.

The quantity $Z$ in Eq. (5) denotes the kernel of the Faddeev equation. It contains the one-particle exchange

\footnotetext{
${ }^{2}$ Namely, in order to regularize this expression, we subtract and add the same expression, where the sum is replaced by an integral. The integral is then calculated within the dimensional regularization.
}

diagram, as well as the local term, corresponding to the particle-dimer interaction (three-particle force). We shall see that, up to and including terms of order $L^{-6}$, it suffices to retain the nonderivative coupling only, which is described by a single constant $H_{0}(\Lambda)$. The kernel then takes the form

$$
Z(\mathbf{p}, \mathbf{q} ; E)=\frac{1}{-m E+\mathbf{p}^{2}+\mathbf{q}^{2}+\mathbf{p q}}+\frac{H_{0}(\Lambda)}{\Lambda^{2}} .
$$

The dependence of $H_{0}(\Lambda)$ on the cutoff is such that the infinite-volume scattering amplitude is cutoff-independent. In a finite volume, this ensures the cutoff-independence of the spectrum.

After giving a short summary of the approach, introduced in Refs. [17,18,20], we next turn to the derivation of the perturbative expansion of the energy level shifts on the basis of the quantization condition. Here, we first note that the equation det $\mathcal{A}=0$, which defines all energies, is very difficult to handle directly. For this reason, we shall first derive the basic matrix identity, which will then allow us to carry out the expansion of the energy shift in powers of $1 / L$. This identity gives the determinant as a product of two factors: the determinant of a matrix with certain row(s)/column(s) erased, and the remainder. The crucial point is that, in the case of free particles, the different rows/columns of the matrix $\mathcal{A}$ correspond to the welldefined free levels. Then, the properly "chopped" determinant in the interacting case should not have a zero, corresponding to a given shifted free level (here, one assumes that the shift is perturbative in $1 / L$ and that it is much smaller than the distance between levels). Consequently, all information about a given level is contained in the remainder, which has a much simpler form and where the unnecessary information about all other levels is already factored out.

In order to demonstrate how this factorization works in practice, consider a generic matrix $\mathcal{A}$ and select an arbitrary value of the index, $r$. Let $\mathcal{A}_{/ p q}$ denote a matrix, which is obtained from $\mathcal{A}$ by crossing out the row $p$ and the column $q$. Expanding the determinant of the matrix $\mathcal{A}$ in cofactors, we get

$$
\operatorname{det} \mathcal{A}=\mathcal{A}_{r r} \operatorname{det} \mathcal{A}_{/ r r}-\sum_{k, l \neq r}(-1)^{k+l} \mathcal{A}_{r k} \mathcal{A}_{l r} \operatorname{det} \mathcal{A}_{/ r k / l r}
$$

Taking into account the fact that $\mathcal{A}_{/ r k / l r}=\mathcal{A}_{/ r r / l k}$ and that the inverse of a matrix $A_{/ r r}$ is equal to its conjugate divided by its determinant:

$$
\left(\mathcal{A}_{/ r r}^{-1}\right)_{k l}=(-1)^{k+l} \operatorname{det} \mathcal{A}_{/ r r / l k}\left(\operatorname{det} \mathcal{A}_{/ r r}\right)^{-1},
$$

we get: 


$$
\operatorname{det} \mathcal{A}=\left(\mathcal{A}_{r r}-\sum_{k, l \neq r} \mathcal{A}_{r k}\left(\mathcal{A}_{/ r r}\right)_{k l}^{-1} \mathcal{A}_{l r}\right) \operatorname{det} \mathcal{A}_{/ r r}
$$

Hence, from the quantization condition $\operatorname{det} \mathcal{A}=0$ it follows that the quantity in brackets, which does not contain determinants anymore, should vanish. ${ }^{3}$

In our case, $\mathcal{A}_{p q}=\left(\tau^{-1}-Z\right)_{p q}=\left(\tau^{-1}[1-\tau Z]\right)_{p q}$. Taking into account the fact that $\tau$ is a diagonal matrix, i.e., $\tau_{p q}=\delta_{p q} \tau_{p}$, we get:

$$
\begin{aligned}
\mathcal{A}_{r r} & =\left(\tau^{-1}-Z\right)_{r r} \\
& =\sum_{k, l \neq r} \mathcal{A}_{r k}\left(\mathcal{A}_{/ r r}\right)_{k l}^{-1} \mathcal{A}_{l r} \\
& =\sum_{k, l \neq r} Z_{r k}(1-\tau Z)_{k l}^{-1} \tau_{l} Z_{l r} \\
& =\sum_{k \neq r} Z_{r k} \tau_{k} Z_{k r}+\sum_{k, l \neq r} Z_{r k} \tau_{k} Z_{k l} \tau_{l} Z_{l r}+\cdots
\end{aligned}
$$

This matrix identity can be used to produce a systematic expansion of the energy shift in powers of $1 / L$, as we shall see in the following. Here, we just note that the r.h.s. of Eq. (13) is nothing but the multiple-scattering expansion of the particle-dimer scattering amplitude in a finite volume, starting from the second term. At the end, everything will sum up in the infinite-volume exact particle-dimer amplitude plus a well-defined set of the finite-volume corrections that emerge from the first few terms in this expansion.

\section{THREE PARTICLE GROUND STATE ENERGY SHIFT}

\section{A. Solving the quantization condition}

In order to calculate the ground state energy shift, we have to single out the matrix element $\mathcal{A}_{r r}$, where $r$ corresponds to the state with $\mathbf{p}=0$. Further, the quantity $E$ in the quantization condition corresponds to the energy above the three free particle threshold, i.e., to the energy shift $\Delta E_{3}(L)$ in this particular case.

It can be seen from Eq. (13) that $E$ is of order $L^{-3}$. We arrive at this conclusion neglecting the right-hand side (r.h.s.) of this equation altogether. It can then be checked a posteriori that taking the r.h.s. into account gives only the $O\left(L^{-1}\right)$ corrections to the leading-order result. Further, since the momenta are of order $L^{-1}$, the Taylor expansion of the energy denominators in powers of $q_{0}^{2}$ is justified and, for the quantity $\tau_{L}^{-1}(\mathbf{0} ; E)$, one obtains

\footnotetext{
${ }^{3}$ Of course, this condition gives only part of the roots of the whole determinant - those, which are not given by the solution of the equation $\operatorname{det} \mathcal{A}_{/ r r}=0$. The root we are interested in-the one that lies in the vicinity of a given unperturbed energy level-is among these roots.
}

$$
\begin{aligned}
8 \pi \tau_{L}^{-1}(\mathbf{0} ; E)= & -\frac{1}{a}+\frac{1}{2} r q_{0}^{2}+\cdots-\frac{4 \pi}{L^{3}} \sum_{\mathbf{l}} \frac{1}{\mathbf{l}^{2}-q_{0}^{2}} \\
= & -\frac{1}{a}+\frac{2 \pi^{2}}{L^{2}} r \kappa^{2}+\frac{1}{\pi L} \frac{1}{\kappa^{2}}-\frac{1}{\pi L} \mathcal{I} \\
& -\frac{1}{\pi L} \kappa^{2} \mathcal{J}-\frac{1}{\pi L} \kappa^{4} \mathcal{K}+\cdots,
\end{aligned}
$$

where $q_{0}^{2}=m E$ and $\kappa=L q_{0} /(2 \pi)$. The constants $\mathcal{I}, \mathcal{J}, \mathcal{K}$ are given in Appendix C, see also Ref. [34]. Recalling that at leading order $q_{0}^{2}=O\left(L^{-3}\right)$ and hence $\kappa^{2}=O\left(L^{-1}\right)$, it is easy to verify that the neglected terms in Eq. (14) are of order of $L^{-4}$ and higher.

The expansion of the quantity $\tau_{L}^{-1}(\mathbf{p} ; E)$ at a nonzero value of the momentum $\mathbf{p}$ can be obtained similarly (one needs only leading terms in this expansion):

$$
8 \pi \tau_{L}^{-1}(\mathbf{p} ; E)=-\frac{1}{a}+\sqrt{\frac{3}{4} \mathbf{p}^{2}}-\frac{1}{\pi L} \tilde{\mathcal{I}}(\mathbf{n})+\cdots,
$$

where $\mathbf{p}=2 \pi \mathbf{n} / L$ and

$\tilde{\mathcal{I}}(\mathbf{n})=\lim _{N \rightarrow \infty}\left\{\sum_{\mathbf{j}}^{N} \frac{1}{\mathbf{j}^{2}+\mathbf{n}^{2}+\mathbf{n} \mathbf{j}}-4 \pi N+2 \pi^{2} \sqrt{\frac{3}{4} \mathbf{n}^{2}}\right\}$.

Note that the above equation, unlike Eq. (14), does not contain singular terms, proportional to $\kappa^{-2}$.

Using Eq. (13) the three-body quantization condition is written as:

$$
8 \pi \tau_{L}^{-1}\left(\mathbf{0} ; \frac{q_{0}^{2}}{m}\right)-\frac{8 \pi}{L^{3}} Z\left(\mathbf{0}, \mathbf{0} ; \frac{q_{0}^{2}}{m}\right)-\frac{8 \pi}{L^{3}} \Delta=0,
$$

where,

$$
\Delta=\sum_{k \neq r} Z_{r k} \tau_{k} Z_{k r}+\sum_{k, l \neq r} Z_{r k} \tau_{k} Z_{k l} \tau_{l} Z_{l r}+\cdots
$$

The quantity $\Delta$ does not contain singular terms either. Expanding in powers of $\kappa^{2}$, we get

$L^{-3} \Delta=\frac{\Delta^{(0)}}{L^{2}}+\frac{\Delta^{(1)}}{L^{3}} \ln \frac{m L}{2 \pi}+\frac{\Delta^{(2)}}{L^{3}}+\frac{\Delta^{(3)}}{L^{2}} \kappa^{2}+O\left(L^{-4}\right)$.

Taking into account Eqs. (9), (14) and (19), the three-body quantization condition, Eq. (17), can be solved with respect to $\kappa^{2}$ by iterations. The result, up to and including terms of order $L^{-6}$, is given by: 


$$
\begin{aligned}
\kappa^{2}= & \frac{3 a}{\pi L}\left\{1-\left(\frac{a}{\pi L}\right) \mathcal{I}+\left(\frac{a}{\pi L}\right)^{2}\left(\mathcal{I}^{2}-3 \mathcal{J}-\frac{(2 \pi)^{3}}{a} \Delta^{(0)}\right)\right. \\
& \left.-\frac{8 \pi a}{L^{3}} \Delta^{(1)} \ln \frac{m L}{2 \pi}+\frac{6 \pi a^{2} r}{L^{3}}+\frac{Y}{L^{3}}\right\},
\end{aligned}
$$

where

$$
\begin{aligned}
Y= & \left(\frac{a}{\pi}\right)^{3}\left(-\mathcal{I}^{3}+9 \mathcal{I} \mathcal{J}-9 \mathcal{K}+\frac{(2 \pi)^{3}}{a} 2 \mathcal{I} \Delta^{(0)}\right) \\
& -8 \pi a\left(\frac{H_{0}(\Lambda)}{\Lambda^{2}}+\Delta^{(2)}+\frac{3 a}{\pi} \Delta^{(3)}\right) .
\end{aligned}
$$

Hence, in order to complete the calculation, it remains to evaluate the quantities $\Delta^{(0)}, \ldots, \Delta^{(3)}$. To this end, let us consider the terms, entering in $\Delta$, separately.

\section{B. One iteration}

The crucial point in the derivation of the energy shift is that the leading-order corrections (in $1 / L$ ) emerge from the first two terms in the expression of $\Delta$. In all other terms, up to and including order $L^{-6}$, one could simply perform the infinite-volume limit, replacing sums by integrals. In the following, we shall illustrate this statement by explicit calculations, writing $\Delta=S_{1}+S_{2}+\cdots$ and starting from the first term in the expansion:

$$
\begin{aligned}
S_{1} & =\sum_{k \neq 0} Z_{0 k} \tau_{k} Z_{k 0} \\
& =\frac{8 \pi}{L^{3}} \sum_{\mathbf{k} \neq \mathbf{0}}^{\Lambda}\left(\frac{1}{\mathbf{k}^{2}-m E}+\frac{H_{0}(\Lambda)}{\Lambda^{2}}\right)^{2} \frac{-a}{1-a \sqrt{\frac{3}{4} \mathbf{k}^{2}}+\frac{a}{\pi L} \tilde{\mathcal{I}}(\mathbf{n})},
\end{aligned}
$$

where $\mathbf{k}=2 \pi \mathbf{n} / L$.

The leading corrections arise from the most infraredsingular terms. In order to single out these corrections, we first note that it is safe to expand the above expression in powers of $q_{0}^{2}=m E$ and $\frac{a}{\pi L} \tilde{\mathcal{I}}(\mathbf{n})$. Namely, the quantity $\tilde{\mathcal{I}}(\mathbf{n})$ is uniformly bound from above for all values of $\mathbf{n}$ (in difference to $\sqrt{\mathbf{k}^{2}}$, which also appears in the denominator). The result of the expansion is given by

$$
\begin{aligned}
S_{1}= & \frac{8 \pi}{L^{3}} \sum_{\mathbf{k} \neq \mathbf{0}}^{\Lambda}\left(\frac{1}{\mathbf{k}^{2}}+\frac{H_{0}(\Lambda)}{\Lambda^{2}}\right)^{2} \frac{-a}{1-a \sqrt{\frac{3}{4} \mathbf{k}^{2}}} \\
& +\frac{8 \pi}{L^{3}} \sum_{\mathbf{k} \neq \mathbf{0}}^{\Lambda} \frac{2 q_{0}^{2}}{\mathbf{k}^{4}}\left(\frac{1}{\mathbf{k}^{2}}+\frac{H_{0}(\Lambda)}{\Lambda^{2}}\right) \frac{-a}{1-a \sqrt{\frac{3}{4} \mathbf{k}^{2}}} \\
& -\frac{8 \pi}{L^{3}} \sum_{\mathbf{k} \neq \mathbf{0}}^{\Lambda} \frac{a \tilde{\mathcal{I}}(\mathbf{n})}{\pi L}\left(\frac{1}{\mathbf{k}^{2}}+\frac{H_{0}(\Lambda)}{\Lambda^{2}}\right)^{2} \frac{-a}{1-a \sqrt{\frac{3}{4} \mathbf{k}^{2}}}+\cdots \\
= & S_{1}^{\prime}+S_{1}^{\prime \prime}+S_{1}^{\prime \prime \prime}+\cdots .
\end{aligned}
$$

Below, we shall consider all these terms separately and start from the first one, writing $S_{1}^{\prime}=\left(S_{1}^{\prime}-\bar{S}_{1}^{\prime}\right)+\bar{S}_{1}^{\prime}$, where $\bar{S}_{1}^{\prime}$ is the most singular piece of $S_{1}^{\prime}$, as $\mathbf{k} \rightarrow 0$ :

$$
\bar{S}_{1}^{\prime}=\frac{8 \pi}{L^{3}} \sum_{\mathbf{k} \neq \mathbf{0}}^{\Lambda} \frac{1}{\mathbf{k}^{4}}(-a)\left\{1+a \sqrt{\frac{3}{4} \mathbf{k}^{2}}\right\} .
$$

It is immediately seen that the difference $\left(S_{1}^{\prime}-\bar{S}_{1}^{\prime}\right)$ goes as $\mathbf{k}^{-2}$, when $\mathbf{k} \rightarrow 0$. Further, for the evaluation of the sums in Eq. (24) we shall use the formulas given in Ref. [8]:

$$
\begin{aligned}
& \frac{1}{L^{3}} \sum_{\mathbf{p}} \frac{f\left(\mathbf{p}^{2}\right)}{\mathbf{p}^{2}}=\int \frac{d^{3} \mathbf{p}}{(2 \pi)^{3}} \frac{f\left(\mathbf{p}^{2}\right)}{\mathbf{p}^{2}}+\frac{\mathcal{I} f(0)}{(2 \pi)^{2} L}-\frac{f^{\prime}(0)}{L^{3}}+\cdots, \\
& \frac{1}{L^{3}} \sum_{\mathbf{p}} \frac{g\left(\mathbf{p}^{2}\right)}{\mathbf{p}^{4}}=\frac{L \mathcal{J} g(0)}{(2 \pi)^{4}}+\int \frac{d^{3} \mathbf{p}}{(2 \pi)^{3}} \frac{g\left(\mathbf{p}^{2}\right)-g(0)}{\mathbf{p}^{4}}+\cdots, \\
& \frac{1}{L^{3}} \sum_{\mathbf{p}} \frac{h\left(\mathbf{p}^{2}\right)}{\mathbf{p}^{6}}=\frac{L^{3} \mathcal{K} h(0)}{(2 \pi)^{4}}+\cdots .
\end{aligned}
$$

The most infrared-singular piece in Eq. (24) is the one containing $1 / \mathbf{k}^{4}$. To calculate this contribution, we shall demonstrate a trick that will be systematically used below:

$$
\begin{aligned}
\frac{1}{L^{3}} \sum_{\mathbf{k} \neq \mathbf{0}}^{\Lambda} \frac{1}{\mathbf{k}^{4}} & =\frac{L \mathcal{J}}{(2 \pi)^{4}}-\lim _{\varepsilon \rightarrow 0} \int_{\Lambda}^{\infty} \frac{d^{3} \mathbf{k}}{(2 \pi)^{3}} \frac{1}{\left(\mathbf{k}^{2}+\varepsilon^{2}\right)^{2}} \\
& =\lim _{\varepsilon \rightarrow 0}\left[\int^{\Lambda} \frac{d^{3} \mathbf{k}}{(2 \pi)^{3}} \frac{1}{\left(\mathbf{k}^{2}+\varepsilon^{2}\right)^{2}}-\frac{1}{8 \pi \varepsilon}\right]+\frac{L \mathcal{J}}{(2 \pi)^{4}} .
\end{aligned}
$$

The physical meaning of introducing the quantity $\varepsilon$ is to shift the total energy of the particle-dimer system $E=$ $-\varepsilon^{2} / m$ slightly below threshold. The particle-dimer amplitude at zero initial and final momenta is singular at threshold corresponding to $\varepsilon=0$. Subtracting the quantity $(8 \pi \varepsilon)^{-1}$ from the amplitude eliminates the singularity, and the limit $\varepsilon \rightarrow 0$ can be performed in the remainder. We shall do the same procedure in all terms in the perturbative series. The key property of these series is that the number of the diagrams, leading to the singular behavior at threshold, is finite, even if the perturbative series for the amplitude contain infinitely many terms. Consequently, one may find all singular terms in a closed form. In a finite volume, exactly these singular diagrams lead to the leading contributions to the energy shift.

In order to demonstrate the above statement, consider, e.g., a less singular term in $S_{1}^{\prime}$, containing one power of the coupling $H_{0}(\Lambda)$ :

$$
\frac{1}{L^{3}} \sum_{\mathbf{k} \neq \mathbf{0}}^{\Lambda} \frac{2}{\mathbf{k}^{2}} \frac{H_{0}(\Lambda)}{\Lambda^{2}}=\lim _{\varepsilon \rightarrow 0} \int^{\Lambda} \frac{d^{3} \mathbf{k}}{(2 \pi)^{3}} \frac{2}{\mathbf{k}^{2}+\varepsilon^{2}} \frac{H_{0}(\Lambda)}{\Lambda^{2}}+\cdots .
$$


This expression is not singular at $\varepsilon \rightarrow 0$ and behaves as $L^{0}$ at large $L$, whereas the integral in Eq. (26) has a singular piece of order $\varepsilon^{-1}$ that translates into an $O(L)$ term at large $L$.

Continuing in the same manner with the expansion in Eq. (26) we calculate the next most singular contribution to Eq. (24):

$$
\begin{aligned}
\frac{1}{L^{3}} \sum_{\mathbf{k} \neq \mathbf{0}}^{\Lambda} \frac{1}{|\mathbf{k}|^{3}} & =\frac{1}{(2 \pi)^{3}}\left(\sum_{\mathbf{j} \neq \mathbf{0}}^{\Lambda L / 2 \pi} \frac{1}{|\mathbf{j}|^{3}}-\int_{1}^{\Lambda L / 2 \pi} \frac{d^{3} \mathbf{j}}{|\mathbf{j}|^{3}}+\int_{1}^{\Lambda L / 2 \pi} \frac{d^{3} \mathbf{j}}{|\mathbf{j}|^{3}}\right) \\
& =\frac{1}{(2 \pi)^{3}}\left(4 \pi \ln \frac{\Lambda L}{2 \pi}+\mathcal{L}+O\left(L^{-1}\right)\right),
\end{aligned}
$$

where the quantity $\mathcal{L}$ is given in Appendix $\mathrm{C}$, see also Appendix B.

Using the same trick, this expression can be rewritten as

$$
\frac{1}{L^{3}} \sum_{\mathbf{k} \neq \mathbf{0}}^{\Lambda} \frac{1}{|\mathbf{k}|^{3}}=\frac{2}{\sqrt{3}} \lim _{\varepsilon \rightarrow 0}\left[\int^{\Lambda} \frac{d^{3} \mathbf{k}}{(2 \pi)^{3}} \frac{1}{\left(\mathbf{k}^{2}+\varepsilon^{2}\right)^{2}} \sqrt{\frac{3}{4} \mathbf{k}^{2}+\varepsilon^{2}}+\frac{\sqrt{3}}{4 \pi^{2}} \ln \frac{\varepsilon}{m}\right]+\frac{1}{2 \pi^{2}} \ln \frac{m L}{2 \pi}+\frac{1}{2 \pi^{2}}\left(\frac{1}{2}(1-\ln 3)+\frac{\sqrt{3} \pi}{18}\right)+\frac{1}{(2 \pi)^{3}} \mathcal{L}+\cdots
$$

There are no more singular terms in $S_{1}^{\prime}$, given by Eq. (24) and, hence, the summation can be directly replaced by the integration in the remaining terms. Further, the quantities $S_{1}^{\prime \prime}$ and $S_{1}^{\prime \prime \prime}$ can be evaluated directly:

$$
S_{1}^{\prime \prime}=-\frac{a q_{0}^{2} L^{3}}{4 \pi^{5}} \mathcal{K}+\cdots, \quad S_{1}^{\prime \prime \prime}=\frac{a^{2}}{2 \pi^{4}} \mathcal{G}+\cdots,
$$

where the ellipses stand for the subleading terms in $L$ and the quantity $\mathcal{G}$ in given in Appendix C, see also Appendix B.

Putting all pieces together, we finally obtain

$$
\begin{aligned}
S_{1}= & \lim _{\varepsilon \rightarrow 0}\left[\int^{\Lambda} \frac{d^{3} \mathbf{k}}{(2 \pi)^{3}}\left(\frac{1}{\mathbf{k}^{2}+\varepsilon^{2}}+\frac{H_{0}(\Lambda)}{\Lambda^{2}}\right)^{2} \frac{-8 \pi a}{1-a \sqrt{\frac{3}{4} \mathbf{k}^{2}+\varepsilon^{2}}}+\frac{a}{\varepsilon}-\frac{2 \sqrt{3} a^{2}}{\pi} \ln \frac{\varepsilon}{m}\right] \\
& -\frac{a L \mathcal{J}}{2 \pi^{3}}-\frac{2 \sqrt{3} a^{2}}{\pi} \ln \frac{m L}{2 \pi}-\frac{2 \sqrt{3} a^{2}}{\pi}\left(\frac{1}{2}(1-\ln 3)+\frac{\sqrt{3} \pi}{18}\right)-\frac{a^{2} \sqrt{3}}{2 \pi^{2}} \mathcal{L}+\frac{a^{2}}{2 \pi^{4}} \mathcal{G}-\frac{a q_{0}^{2} L^{3}}{4 \pi^{5}} \mathcal{K}+\cdots
\end{aligned}
$$

Note that the divergence-subtracted threshold particle-dimer amplitude appears in the first line of this equation.

\section{Two iterations}

The method, used above, can be applied to the second term in $\Delta$, which is written down as:

$$
S_{2}=\frac{(8 \pi)^{2}}{L^{6}} \sum_{\mathbf{k} \neq \mathbf{0}}^{\Lambda} \sum_{\mathbf{q} \neq \mathbf{0}}^{\Lambda}\left(\frac{1}{\mathbf{k}^{2}}+\frac{H_{0}(\Lambda)}{\Lambda^{2}}\right) \frac{-a}{1-a \sqrt{\frac{3}{4} \mathbf{k}^{2}}}\left(\frac{1}{\mathbf{k}^{2}+\mathbf{q}^{2}+\mathbf{k q}}+\frac{H_{0}(\Lambda)}{\Lambda^{2}}\right) \frac{-a}{1-a \sqrt{\frac{3}{4} \mathbf{q}^{2}}}\left(\frac{1}{\mathbf{q}^{2}}+\frac{H_{0}(\Lambda)}{\Lambda^{2}}\right) .
$$

Note that here we consistently drop the terms proportional to $q_{0}^{2}$ and $\tilde{\mathcal{I}}(\mathbf{n})$, since these do not contribute at the accuracy we are working. There is only one singular diagram at this order $S_{2}=\left(S_{2}-\bar{S}_{2}\right)+\bar{S}_{2}$, where

$$
\bar{S}_{2}=a^{2} \frac{(8 \pi)^{2}}{L^{6}} \sum_{\mathbf{k} \neq \mathbf{0}}^{\Lambda} \sum_{\mathbf{q} \neq \mathbf{0}}^{\Lambda} \frac{1}{\mathbf{k}^{2}} \frac{1}{\mathbf{k}^{2}+\mathbf{q}^{2}+\mathbf{k q}} \frac{1}{\mathbf{q}^{2}}=\frac{a^{2}}{\pi^{4}}\left\{\frac{8 \pi^{4}}{3} \ln \frac{\Lambda L}{2 \pi}+2 \mathcal{Q}\right\}
$$

where the quantity $\mathcal{Q}$ is given in Appendix C (see Appendix B for the derivation of this formula). 
On the other hand, we can use our trick again and calculate the integral

$$
I_{\varepsilon}=a^{2} \int^{\Lambda} \frac{d^{3} \mathbf{k}}{(2 \pi)^{3}} \int^{\Lambda} \frac{d^{3} \mathbf{q}}{(2 \pi)^{3}} \frac{1}{\mathbf{k}^{2}+\varepsilon^{2}} \frac{1}{\mathbf{k}^{2}+\mathbf{q}^{2}+\mathbf{k q}+\varepsilon^{2}} \frac{1}{\mathbf{q}^{2}+\varepsilon^{2}}=\frac{a^{2}}{24 \pi^{2}} \ln \frac{\Lambda}{\varepsilon}+\frac{a^{2}}{(2 \pi)^{6}} \mathcal{I}_{0},
$$

where $\mathcal{I}_{0} \simeq-375.754658$ denotes a numerical constant. The derivation of the above formula, as well as an explicit expression for this constant is contained in Appendix B. Since all other terms in the expansion of $S_{2}$, given by the Eq. (32), are nonsingular, one immediately gets

$$
\begin{aligned}
S_{2}= & \lim _{\varepsilon \rightarrow 0}\left[\int \frac{d^{3} \mathbf{k}}{(2 \pi)^{3}} \int^{\Lambda} \frac{d^{3} \mathbf{q}}{(2 \pi)^{3}}\left(\frac{1}{\mathbf{k}^{2}+\varepsilon^{2}}+\frac{H_{0}(\Lambda)}{\Lambda^{2}}\right) \frac{-8 \pi a}{1-a \sqrt{\frac{3}{4} \mathbf{k}^{2}+\varepsilon^{2}}}\right. \\
& \left.\times\left(\frac{1}{\mathbf{k}^{2}+\mathbf{q}^{2}+\mathbf{k} \mathbf{q}+\varepsilon^{2}}+\frac{H_{0}(\Lambda)}{\Lambda^{2}}\right) \frac{-8 \pi a}{1-a \sqrt{\frac{3}{4} \mathbf{q}^{2}+\varepsilon^{2}}}\left(\frac{1}{\mathbf{q}^{2}+\varepsilon^{2}}+\frac{H_{0}(\Lambda)}{\Lambda^{2}}\right)+\frac{8 a^{2}}{3} \ln \frac{\varepsilon}{m}\right] \\
& +\frac{8 a^{2}}{3} \ln \frac{m L}{2 \pi}+\frac{a^{2}}{\pi^{4}}\left(2 \mathcal{Q}-\mathcal{I}_{0}\right) .
\end{aligned}
$$

Note that there are no singular terms at higher orders in the iteration series, so one can stop here and write down an expression for the quantity $\Delta$ defined in Eq. (19).

\section{An expression for the quantity $\Delta$}

In order to write down a closed expression for the quantity $\Delta$, it is convenient to define the divergencesubtracted threshold particle-dimer amplitude in the following manner:

(1) Consider the particle-dimer scattering amplitude $\mathcal{M}\left(\mathbf{0}, \mathbf{0} ;-\frac{\varepsilon^{2}}{m}\right)$, which is a solution of the Faddeev equation in the infinite volume with the kernel given by $Z$.

(2) Taking into account that $Z\left(\mathbf{0}, \mathbf{0} ;-\frac{\varepsilon^{2}}{m}\right)=\frac{1}{\varepsilon^{2}}+\frac{H_{0}(\Lambda)}{\Lambda^{2}}$, see Eq. (9), one can define the divergence-subtracted amplitude $\hat{\mathcal{M}}$ as

$$
\begin{aligned}
& \mathcal{M}\left(\mathbf{0}, \mathbf{0} ;-\frac{\varepsilon^{2}}{m}\right) \\
& =\frac{\mathcal{M}_{-2}}{\varepsilon^{2}}+\frac{\mathcal{M}_{-1}}{\varepsilon}+\mathcal{M}_{0} \ln \frac{\varepsilon}{m}+\hat{\mathcal{M}}+\cdots,
\end{aligned}
$$

where the coefficients $\mathcal{M}_{-2}, \mathcal{M}_{-1}, \mathcal{M}_{0}$ are adjusted so that $\hat{\mathcal{M}}$ does not depend on $\varepsilon$ in the limit $\varepsilon \rightarrow 0$, and the ellipses stand for the terms which vanish in this limit.

Now, we can easily read off the expressions for $\Delta^{(0)}, \ldots, \Delta^{(3)}$ from the above formulas:

$$
\begin{aligned}
\Delta^{(0)} & =-\frac{a \mathcal{J}}{2 \pi^{3}}, \quad \Delta^{(1)}=-\frac{2 \sqrt{3} a^{2}}{\pi}+\frac{8 a^{2}}{3}, \\
\frac{H_{0}(\Lambda)}{\Lambda^{2}}+\Delta^{(2)} & =\hat{\mathcal{M}}+\frac{a^{2}}{(2 \pi)^{3}} \mathcal{B}, \quad \Delta^{(3)}=-\frac{a}{\pi^{3}} \mathcal{K},
\end{aligned}
$$

where $\mathcal{B}$ is a combination of many numerical factors, emerging in different equations:

$$
\begin{aligned}
\mathcal{B}= & -16 \pi^{2} \sqrt{3}\left(\frac{1}{2}(1-\ln 3)+\frac{\sqrt{3} \pi}{18}\right) \\
& -4 \pi \sqrt{3} \mathcal{L}+\frac{4}{\pi} \mathcal{G}+\frac{8}{\pi}\left(2 \mathcal{Q}-\mathcal{I}_{0}\right) .
\end{aligned}
$$

\section{E. Final expression for the ground-state energy shift at $O\left(L^{-6}\right)$}

After substituting into Eqs. (20) and (21), we finally get

$$
\begin{aligned}
\kappa^{2}= & \frac{3 a}{\pi L}\left\{1-\left(\frac{a}{\pi L}\right) \mathcal{I}+\left(\frac{a}{\pi L}\right)^{2}\left(\mathcal{I}^{2}+\mathcal{J}\right)\right. \\
& \left.+\left(\frac{a}{\pi L}\right)^{3} 16 \pi^{3}\left(\sqrt{3}-\frac{4 \pi}{3}\right) \ln \frac{m L}{2 \pi}+\frac{6 \pi a^{2} r}{L^{3}}+\frac{Y}{L^{3}}\right\},
\end{aligned}
$$

where

$$
Y=\left(\frac{a}{\pi}\right)^{3}\left(-\mathcal{I}^{3}+\mathcal{I} \mathcal{J}+15 \mathcal{K}-\pi \mathcal{B}\right)-8 \pi a \hat{\mathcal{M}}
$$

In order to simplify the comparison of our result with the ones from the literature, it is convenient to introduce the notation $\mathcal{R}=\left(\mathcal{G}-\pi^{2} \sqrt{3} \mathcal{L}\right) / 2 \simeq-32.60560475$. Our result can be then rewritten as

$$
\begin{aligned}
Y= & \left(\frac{a}{\pi}\right)^{3}\left(-\mathcal{I}^{3}+\mathcal{I} \mathcal{J}+15 \mathcal{K}-8(2 \mathcal{Q}+\mathcal{R})\right) \\
& -8 \pi a\left(\hat{\mathcal{M}}+\frac{a^{2}}{8 \pi^{3}} \delta\right),
\end{aligned}
$$


where the quantity

$\delta=-16 \pi^{2} \sqrt{3}\left(\frac{1}{2}(1-\ln 3)+\frac{\sqrt{3} \pi}{18}\right)-\frac{8}{\pi} \mathcal{I}_{0} \simeq 887.65392$

contains only infinite-volume integrals.

\section{SHIFT OF THE LOWEST PARTICLE-DIMER SCATTERING LEVEL}

If the two-body scattering length is positive and large, there exists a shallow two-body bound state (a dimer). The set of the asymptotic states in the three-body problem in the infinite volume then includes the states, describing a dimer and a spectator, separated by a large distance and do not interact with each other. The energy of such an asymptotic state is $E=E_{d}+\frac{\mathbf{p}^{2}}{2\left(2 m+E_{d}\right)}+\frac{\mathbf{p}^{2}}{2 m}$, where $2 m+E_{d}$ is the mass of a dimer is the rest frame and $\mathbf{p}$ is the momentum of the spectator in the rest frame of three particles. In case of a large scattering length, we have $E_{d}=-1 /\left(m a^{2}\right)+\cdots$, where the ellipses stand for the terms emerging from higher orders in the effective range expansion. One expects that these infinite-volume scattering levels obtain shifts in a finite volume, just similar to what the three-particle levels do. It will be seen below that our quantization condition allows one to calculate these shifts as well.

In this paper, we restrict ourselves to the calculation of the shift of the particle-dimer ground state at order $L^{-3}$. This corresponds to the spectator momentum $\mathbf{p}=0$. The energy of the state is given by $E=E_{d}+\Delta_{d}$, where $\Delta_{d}$ denotes the finite-volume shift. The quantization condition is given by, cf. Eq. (17):

$$
8 \pi \tau_{L}^{-1}(\mathbf{0} ; E)-\frac{8 \pi}{L^{3}} Z(\mathbf{0}, \mathbf{0} ; E)-\frac{8 \pi}{L^{3}} \Delta(\mathbf{0}, \mathbf{0} ; E)=0,
$$

where the quantity $\Delta$ contains the terms with multiple iterations, see the previous section. Further, since $E_{d}$ is below the two-particle threshold,

$$
8 \pi \tau_{L}^{-1}(\mathbf{0} ; E)=8 \pi K^{-1}(\mathbf{0} ; E)+\sqrt{-m E}+\cdots,
$$

where the ellipses stand for the exponentially suppressed terms and

$$
8 \pi K^{-1}(\mathbf{0} ; E)=-\frac{1}{a}+\frac{1}{2} r(m E)+\cdots
$$

denotes the infinite-volume two-body $K$-matrix in the rest frame.

Solving the quantization condition for $\Delta_{d}$ iteratively, one can replace $E$ by $E_{d}$ in $Z(E)+\Delta(E)$, arriving at the threshold amplitude for the particle-dimer scattering

$$
\mathcal{M}_{d}=Z\left(\mathbf{0}, \mathbf{0} ; E_{d}\right)+\Delta\left(\mathbf{0}, \mathbf{0} ; E_{d}\right) .
$$

Note that, in contrast to the threshold amplitude, introduced in the previous section, this quantity does not contain infrared singularities, since $E_{d} \neq 0$ from the beginning. The solution of the quantization condition to the leading order is given by

$$
\Delta_{d}=\frac{\mathcal{Z}}{L^{3}} \mathcal{M}_{d}
$$

where

$$
\begin{aligned}
\mathcal{Z} & =\frac{-8 \pi}{\frac{m}{2 \sqrt{-m E_{d}}}-\left(8 \pi K^{-1}\right)^{\prime}}, \\
\left(8 \pi K^{-1}\right)^{\prime} & =\left.\frac{d}{d E}\left(8 \pi K^{-1}(\mathbf{0} ; E)\right)\right|_{E=E_{d}} .
\end{aligned}
$$

It is straightforwardly seen that the quantity $\mathcal{Z}$ coincides with the wave function renormalization constant for the dimer field. Indeed, in the normalization, used in this paper, the two-point function of the dimer field is given by ${ }^{4}$

$D(E, \mathbf{0})=\frac{8 \pi}{8 \pi K^{-1}(E)-\sqrt{-m E-i \varepsilon}} \rightarrow \frac{\mathcal{Z}}{E_{d}-E}+\cdots$.

Consequently, the quantity $\mathcal{A}_{d}=\mathcal{Z} \mathcal{M}_{d}$ is just the particledimer scattering amplitude at threshold, and Eq. (47) can be rewritten as

$$
\Delta_{d}=\frac{1}{L^{3}} \mathcal{A}_{d},
$$

which exactly reproduces the two-particle Lüscher equation (1), where the dimer is considered as an elementary particle. This result, of course, does not come as a surprise. In fact, if a dimer is deeply bound and thus can be considered as elementary, the Lüscher formula will hold in higher orders in $1 / L$ as well. The particle-dimer scattering length, the effective range, etc., which appear in this formula, are then related to the parameters of the underlying theory in the same way, as in the infinite volume. However, the case of a loosely bound dimer is less trivial. The leading term in $1 / L$ expansion is still given by the Lüscher formula, but the subleading terms will

\footnotetext{
${ }^{4}$ The normalization of the dimer propagator can be easily read off from Eqs. (329) and (333) of Ref. [36], where the effectiverange expansion in the two-body interaction is carried out at leading order only. Namely, comparing Eq. (333) from that paper with our Eq. (9), one sees that the overall normalization of the particle-dimer amplitudes differs by a factor $g_{2}^{2} / 4$, where $g_{2}$ denotes the leading-order two-body coupling. This fixes the normalization in Eq. (49), where exactly the same multiplicative factor has to be removed, as compared to Eq. (329) of Ref. [36].
} 
contain corrections, which can be attributed to its finite spatial extension. The calculation of these corrections is, however, beyond the scope of the present paper.

The main difference of the particle-dimer energy shift $\Delta_{d}$ with the shift of the three-particle state is that here the quantity $\mathcal{M}_{d}$ appears at leading order already. Hence, the particle-dimer shift will be more sensitive to the value of the three-body coupling $H_{0}(\Lambda)$. This fact can be used for the extraction of the latter quantity from the lattice data.

\section{THE SHIFT OF THE EXCITED THREE-PARTICLE LEVEL}

\section{A. Singular contributions}

The calculation of the shift of the excited levels proceeds along the similar path as for the ground state level, but some modifications are needed. In order to understand the origin of the emerging problems, consider, for instance, the shift of the unperturbed level with the particles having momenta $\mathbf{p}_{1}=(0,0,1), \mathbf{p}_{2}=(0,0,-1)$ and $\mathbf{p}_{3}=(0,0,0)$ (all momenta are given in units of $2 \pi / L)$. There is no unique assignment for the spectator momentum in this case: the magnitude of the spectator momenta can be equal to either 0 or 1 . Let us recall now that, in case of the ground state, the matrix $\mathcal{A}$, considered in Sec. II, contained singular contributions, proportional to $1 / \kappa^{2}$, only in the diagonal matrix element $\mathcal{A}_{r r}$, corresponding to the spectator momentum $|\mathbf{p}|=0$. The quantization condition could be then readily rewritten in form of Eq. (13). In case of the excited levels the situation is different-now, the singular terms are contained in a block, which corresponds to the different assignments of the spectator momenta. In the case, considered above, this will be a $2 \times 2$ block, whose entries correspond to momenta $|\mathbf{p}|=0,1$. Hence, the technique, described in Sec. II, needs to be amended.

For simplicity, we explicitly describe the calculations for the first excited three-particle level, where the momenta are chosen as above. The technique is, however, completely general and can be applied for higher excited states as well. Moreover, already the first excited level will be split between different irreps of the octahedral group, which was not the case for the ground-state- there, the shifted level emerged in the irrep $A_{1}^{+}$only. Hence, one has to project the quantization condition onto the different irreps of the octahedral groups as described in Ref. [20]. Here, we use the notations and terminology from that paper. In particular, we refer to the set of lattice momenta, which can be obtained from a single reference momentum $\mathbf{p}_{0}$ by applying the elements of the octahedral group $\mathbf{p}=g \mathbf{p}_{0}$, $g \in O_{h}$, as to shells. In particular, $|\mathbf{p}|=0$ and $|\mathbf{p}|=1$ correspond to the shells $r=1$ and $r=2$, respectively. The quantization condition, projected onto the irrep $\Gamma$, takes the form $\operatorname{det}\left(8 \pi \tau_{L}^{-1}(r ; E) \delta_{r s} \delta_{\sigma \rho}-\frac{8 \pi \theta^{1 / 2}(r) \theta^{1 / 2}(s)}{G L^{3}} Z_{\rho \sigma}^{\Gamma}(r, s ; E)\right)=0$,

where $r, s$ number the shells, $\sigma, \rho$ label the basis vectors in the irrep $\Gamma, \theta(s)$ is the multiplicity of a given shell (the number of the independent vectors in this shell), $G=48$ is the total number of elements in $O_{h}$ and

$$
Z_{\rho \sigma}^{\Gamma}(r, s ; E)=\sum_{g \in O_{h}}\left(T_{\sigma \rho}^{\Gamma}(g)\right)^{*} Z\left(g \mathbf{p}_{0}(r), \mathbf{k}_{0}(s) ; E\right)
$$

is the projection of the driving term $Z$ onto the irrep $\Gamma$. Here, the set of matrices $T_{\sigma \rho}^{\Gamma}(g)$ forms an irreducible matrix representation $\Gamma$ of the octahedral group $O_{h}$.

Below, we shall first derive the expression for the energy shift for the irrep $A_{1}^{+}$. This irrep is one-dimensional and therefore one may suppress the indices $\sigma, \rho$. Other irreps will be considered later. The expansion for the quantity $\tau_{L}^{-1}$ on the first shell, corresponding to $\mathbf{p}=0$, is given by [cf. with Eq. (14)]:

$$
\begin{aligned}
8 \pi \tau_{L}^{-1}(1 ; E)= & -\frac{1}{a}+\frac{1}{2} r q_{0}^{2}+\cdots-\frac{4 \pi}{L^{3}} \sum_{\mathbf{l}} \frac{1}{\mathbf{l}^{2}-q_{0}^{2}} \\
= & -\frac{1}{a}+\frac{2 \pi^{2}}{L^{2}} r \kappa^{2}+\cdots-\frac{6}{\pi L} \frac{1}{1-\kappa^{2}}-\frac{1}{\pi L} \mathcal{I}_{1} \\
& +\frac{1}{\pi L} \mathcal{J}_{1}\left(1-\kappa^{2}\right)-\frac{1}{\pi L} \mathcal{K}_{1}\left(1-\kappa^{2}\right)^{2}+\cdots,
\end{aligned}
$$

where $\kappa^{2}=1$ corresponds to the unperturbed first excited level. Further, the numerical factors $\mathcal{I}_{1}, \mathcal{J}_{1}, \mathcal{K}_{1}$ are given in Appendix C.

For the expansion on the second shell, let us choose the reference momentum $\mathbf{p}=\frac{2 \pi}{L} \mathbf{j}_{0}$ with $\mathbf{j}_{0}=(0,0,1)$. We get:

$$
\begin{aligned}
8 \pi \tau_{L}^{-1}(2 ; E)= & -\frac{1}{a}+\frac{1}{2} r\left(q_{0}^{2}-\frac{3}{4} \mathbf{p}^{2}\right)+\cdots \\
& -\frac{4 \pi}{L^{3}} \sum_{\mathbf{l}} \frac{1}{\mathbf{l}^{2}+\mathbf{p l}+\mathbf{p}^{2}-q_{0}^{2}} \\
= & -\frac{1}{a}+\frac{2 \pi^{2}}{L^{2}} r\left(\kappa^{2}-\frac{3}{4}\right)+\cdots-\frac{2}{\pi L} \frac{1}{1-\kappa^{2}} \\
& -\frac{1}{\pi L} \mathcal{I}_{2}+\frac{1}{\pi L} \mathcal{J}_{2}\left(1-\kappa^{2}\right) \\
& -\frac{1}{\pi L} \mathcal{K}_{2}\left(1-\kappa^{2}\right)^{2}+\cdots,
\end{aligned}
$$

where $\mathcal{I}_{2}, \mathcal{J}_{2}, \mathcal{K}_{2}$ are defined in Appendix C.

The expansion on the higher shells does not contain singular terms, proportional to $\left(1-\kappa^{2}\right)^{-1}$. Hence, it suffices to retain fewer terms in this expansion for $s>2$ : 


$$
8 \pi \tau_{L}^{-1}(s ; E)=-\frac{1}{a}+\sqrt{\frac{3}{4} \mathbf{p}^{2}-m E}+\cdots-\frac{1}{\pi L} \mathcal{I}\left(\mathbf{j}_{S}\right),
$$

where

$$
\begin{aligned}
\mathcal{I}\left(\mathbf{j}_{s}\right)= & \lim _{N \rightarrow \infty}\left(\sum_{\mathbf{n}}^{N} \frac{1}{\mathbf{n}^{2}+\mathbf{n} \mathbf{j}_{s}+\mathbf{j}_{s}^{2}-1}-4 \pi N\right. \\
& \left.+2 \pi^{2} \sqrt{\frac{3}{4} \mathbf{j}_{s}^{2}-1}\right)
\end{aligned}
$$

and the reference momentum on the shell $s$ is given by $\mathbf{p}=\frac{2 \pi}{L} \mathbf{j}_{s}$.

Next, we consider the projection of the driving term $Z$ onto the irrep $A_{1}^{+}$. This is a trivial representation $T(g)=1$, $g \in O_{h}$. The projection then yields:

$$
\begin{aligned}
\frac{8 \pi \theta(1)}{G L^{3}} \sum_{g \in O_{h}} Z(g \mathbf{0}, \mathbf{0} ; E) & =\frac{2}{\pi L} \frac{1}{-\kappa^{2}}+\frac{8 \pi}{L^{3}} \frac{H_{0}(\Lambda)}{\Lambda^{2}}, \\
\frac{8 \pi \sqrt{\theta(1) \theta(2)}}{G L^{3}} \sum_{g \in O_{h}} Z(g \mathbf{0}, \mathbf{q} ; E) & =\frac{2 \sqrt{6}}{\pi L} \frac{1}{1-\kappa^{2}}+\frac{8 \sqrt{6} \pi H_{0}(\Lambda)}{L^{3}} \frac{1}{\Lambda^{2}}, \\
\frac{8 \pi \theta(2)}{G L^{3}} \sum_{g \in O_{h}} Z(g \mathbf{p}, \mathbf{q} ; E)= & \frac{2}{\pi L} \frac{1}{1-\kappa^{2}}+\frac{8}{\pi L} \frac{1}{2-\kappa^{2}} \\
& +\frac{2}{\pi L} \frac{1}{3-\kappa^{2}}+\frac{48 \pi \frac{H_{0}(\Lambda)}{L^{3}}}{\Lambda^{2}} .
\end{aligned}
$$

Here, $\mathbf{p}, \mathbf{q}$ denote two arbitrary vectors on the shell $s=2$. Like the quantity $\tau_{L}^{-1}$, the projected quantity $Z$ on higher shells does not develop singularities, as expected. Thus, an explicit projection of $Z$ will not be needed for these shells.

\section{B. Solving the quantization condition for the excited states}

In the ground state, only the diagonal matrix element of $\mathcal{A}$, corresponding to the shell $s=1$, contained a singularity. One could then directly apply the method described in Sec. II. However, as we have seen, the structure of the matrix $\mathcal{A}$ in the vicinity of the free excited state energy is different, namely

$$
\mathcal{A}=\left(\begin{array}{cc|cc}
\mathcal{A}_{11} & \mathcal{A}_{12} & \mathcal{A}_{13} & \cdots \\
\mathcal{A}_{21} & \mathcal{A}_{22} & \mathcal{A}_{23} & \ldots \\
\hline \mathcal{A}_{31} & \mathcal{A}_{32} & \mathcal{A}_{33} & \cdots \\
\cdots & \ldots & \ldots & \ldots
\end{array}\right)
$$

The singularities are contained in the upper left corner:

$$
\begin{aligned}
& \mathcal{A}_{11}=-\frac{6}{\pi L} \frac{1}{1-\kappa^{2}}-\frac{1}{a}+\overline{\mathcal{A}}_{11}, \\
& \mathcal{A}_{12}=\mathcal{A}_{21}=-\frac{2 \sqrt{6}}{\pi L} \frac{1}{1-\kappa^{2}}+\overline{\mathcal{A}}_{12}, \\
& \mathcal{A}_{22}=-\frac{4}{\pi L} \frac{1}{1-\kappa^{2}}-\frac{1}{a}+\overline{\mathcal{A}}_{22} .
\end{aligned}
$$

The rest of the matrix elements are regular. Note that, for further convenience, we have explicitly singled out the leading term $1 / a$ in the effective-range expansion as well.

In order to find the energy shift to leading order, it suffices to diagonalize the singular part of the matrix. It is easily seen that this can be achieved through the orthogonal transformation $\mathcal{A} \rightarrow \tilde{\mathcal{O}} \mathcal{A} \tilde{\mathcal{O}}^{T}$, where

$$
\tilde{\mathcal{O}}=\left(\begin{array}{cc|cc}
c & s & 0 & \cdots \\
-s & c & 0 & \cdots \\
\hline 0 & 0 & 1 & \cdots \\
\cdots & \cdots & \cdots & \cdots
\end{array}\right), \quad c=\sqrt{\frac{3}{5}}, \quad s=\sqrt{\frac{2}{5}}
$$

Applying the transformation, we get

$$
\tilde{\mathcal{O}} \mathcal{A} \tilde{\mathcal{O}}^{T}=\left(\begin{array}{cc|c}
X+\cdots & \cdots & \cdots \\
\cdots & -\frac{1}{a}+\cdots & \cdots \\
\hline \cdots & \cdots & \cdots
\end{array}\right)
$$

where we have introduced the notation

$$
X=-\frac{10}{\pi L} \frac{1}{1-\kappa^{2}}-\frac{1}{a}
$$

and the ellipses stand for the regular terms at $\kappa^{2} \rightarrow 1$. It is now clear that, at lowest order, the quantization condition reduces to $X=0$ that gives:

$$
\kappa^{2}-1=\frac{10 a}{\pi L}+O\left(L^{-2}\right)
$$

As seen from Eq. (61), the $2 \times 2$ symmetric matrix, containing only singular terms, has only one nonzero eigenvalue and hence, there exists an unique solution of the quantization condition ${ }^{5}$ in the vicinity of $\kappa^{2}=1$, given by Eq. (63). Unfortunately, at higher orders, the expressions become quite cumbersome and a slightly different technique should be used. With a trial and error method, we

\footnotetext{
${ }^{5}$ Note that this property may not hold in higher excited states, if a given irrep $\Gamma$ appears multiple times in the (generally reducible) representation of the group $O_{h}$ in the vector space, spanned by the individual momenta of three particles. Then, a given unperturbed level may split.
} 
found it convenient to transform the matrix $\mathcal{A}$ by using a different matrix $\mathcal{O}$, where

$$
\mathcal{O}=\left(\begin{array}{cc|cc}
c & s & 0 & \cdots \\
0 & 1 & 0 & \cdots \\
\hline 0 & 0 & 1 & \cdots \\
\cdots & \cdots & \cdots & \cdots
\end{array}\right)
$$

Note that $\mathcal{O}$ is not an orthogonal matrix.

The transformed matrix $\mathcal{O} \mathcal{A O}^{T}$ takes the form

$\mathcal{O} \mathcal{A O}^{T}=\left(\begin{array}{cc|cc}X+\overline{\mathcal{A}}_{11}^{\prime} & s X+\overline{\mathcal{A}}_{12}^{\prime} & \mathcal{A}_{13}^{\prime} & \cdots \\ s X+\overline{\mathcal{A}}_{21}^{\prime} & s^{2} X-c^{2} / a+\overline{\mathcal{A}}_{22} & \mathcal{A}_{23} & \cdots \\ \hline \mathcal{A}_{31}^{\prime} & \mathcal{A}_{32} & \mathcal{A}_{33} & \cdots \\ \cdots & \cdots & \cdots & \cdots\end{array}\right)$

where the "primed" matrix elements are given by:

$$
\begin{aligned}
& \overline{\mathcal{A}}_{11}^{\prime}=c^{2} \overline{\mathcal{A}}_{11}+2 c s \overline{\mathcal{A}}_{12}+s^{2} \overline{\mathcal{A}}_{22}, \\
& \overline{\mathcal{A}}_{12}^{\prime}=\overline{\mathcal{A}}_{21}^{\prime}=c \overline{\mathcal{A}}_{12}+s \overline{\mathcal{A}}_{22}, \\
& \mathcal{A}_{1 i}^{\prime}=\mathcal{A}_{i 1}^{\prime}=c \mathcal{A}_{1 i}+s \mathcal{A}_{2 i}, \quad i=2, \cdots
\end{aligned}
$$

We now may use Eq. (13) to obtain

$$
X+\overline{\mathcal{A}}_{11}^{\prime}=\sum_{k \neq 1} \mathcal{A}_{1 k}^{\prime} \tau_{k} \mathcal{A}_{k 1}^{\prime}+\sum_{k, l \neq 1} \mathcal{A}_{1 k}^{\prime} \tau_{k} Z_{k l} \tau_{l} \mathcal{A}_{k 1}^{\prime}+\cdots
$$

In order to single out all singular terms, we further define

$$
\begin{aligned}
& \mathcal{M}_{k l}=Z_{k l}+\sum_{m \neq 1} Z_{k m} \tau_{m} \mathcal{M}_{m l}, \quad k, l=2, \cdots, \\
& \hat{\mathcal{M}}_{k l}=Z_{k l}+\sum_{m \neq 1,2} Z_{k m} \tau_{m} \hat{\mathcal{M}}_{m l} .
\end{aligned}
$$

From the above equations, it is straightforward to obtain:

$$
\mathcal{M}_{k l}=\hat{\mathcal{M}}_{k l}+\hat{\mathcal{M}}_{k 2} \frac{1}{\tau_{2}^{-1}-\hat{\mathcal{M}}_{22}} \hat{\mathcal{M}}_{2 l}
$$

The goal is achieved because only the quantities $\tau_{2}^{-1}, \hat{\mathcal{M}}_{22}$ contain singularities and everything else is free from singularities. Further, it can be checked that the infinite sum in Eq. (67) can be rewritten in terms of the matrix $\hat{\mathcal{M}}_{k l}$ :

$$
\begin{aligned}
& \sum_{k \neq 1} \mathcal{A}_{1 k}^{\prime} \tau_{k} \mathcal{A}_{k 1}^{\prime}+\sum_{k, l \neq 1} \mathcal{A}_{1 k}^{\prime} \tau_{k} Z_{k l} \tau_{l} \mathcal{A}_{l 1}^{\prime}+\cdots \\
& =\frac{\left(\mathcal{A}_{12}^{\prime}-c \bar{\Delta}_{12}-s \bar{\Delta}_{22}\right)^{2}}{\tau_{2}^{-1}-\hat{\mathcal{M}}_{22}}+c^{2} \bar{\Delta}_{11}+2 c s \bar{\Delta}_{12}+s^{2} \bar{\Delta}_{22}
\end{aligned}
$$

where the quantity

$$
\bar{\Delta}_{k l}=\hat{\mathcal{M}}_{k l}-Z_{k l}
$$

does not contain singular terms.

Now, it is straightforward to obtain:

$$
\begin{aligned}
\tau_{2}^{-1}-\hat{\mathcal{M}}_{22} & =s^{2} X-\frac{c^{2}}{a}+\overline{\mathcal{A}}_{22}-\bar{\Delta}_{22}, \\
\mathcal{A}_{12}^{\prime} & =s X+c \overline{\mathcal{A}}_{12}+s \overline{\mathcal{A}}_{22} .
\end{aligned}
$$

The quantization condition, Eq. (67), now takes the form

$$
\begin{aligned}
X & =\frac{\left(s X+c u_{12}+s u_{22}\right)^{2}}{s^{2} X-\frac{c^{2}}{a}+u_{22}}-\left(c^{2} u_{11}+2 c s u_{12}+s^{2} u_{22}\right), \\
u_{k l} & =\overline{\mathcal{A}}_{k l}-\bar{\Delta}_{k l} .
\end{aligned}
$$

Solving this equation with respect to $X$ finally gives:

$X=-\frac{\left(c^{2} u_{11}+2 c s u_{12}+s^{2} u_{22}\right)-a\left(u_{11} u_{22}-u_{12}^{2}\right)}{1-a\left(c^{2} u_{22}-2 c s u_{12}+s^{2} u_{11}\right)}$.

At the end of this rather lengthy derivation, we have achieved our goal: all singular terms are contained in the quantity $X$, whereas the quantities $\overline{\mathcal{A}}_{k l}$ and $\bar{\Delta}_{k l}$ (and, hence, $u_{k l}$ ), are nonsingular. The expansion for these quantities is given by [cf. with Eq. (19)]:

$\bar{\Delta}_{k l}=\frac{\bar{\Delta}_{k l}^{(0)}}{L^{2}}+\frac{\bar{\Delta}_{k l}^{(1)}}{L^{3}} \ln \frac{m L}{2 \pi}+\frac{\bar{\Delta}_{k l}^{(2)}}{L^{3}}+\frac{\bar{\Delta}_{k l}^{(3)}}{L^{2}}\left(\kappa^{2}-1\right)+\cdots$.

Hence, the expansion of the quantity $\bar{\Delta}_{k l}$ starts at order $L^{-2}$. On the other hand, the expansion of $\overline{\mathcal{A}}_{k l}$ can be read off from Eqs. (53), (54) and (57)—it starts at order $L^{-1}$ :

$$
\begin{aligned}
\overline{\mathcal{A}}_{k l}= & \left(\frac{a_{k l}^{(0)}}{L}+\frac{b_{k l}^{(0)}}{L^{2}}\right)+\left(\frac{a_{k l}^{(1)}}{L}+\frac{b_{k l}^{(1)}}{L^{2}}\right)\left(1-\kappa^{2}\right) \\
& +\frac{a_{k l}^{(2)}}{L}\left(1-\kappa^{2}\right)^{2}+\frac{c_{k l}}{L^{3}} \cdots
\end{aligned}
$$

Since $u_{k l}=O\left(L^{-1}\right)$, the right-hand side of Eq. (74) can be expanded. We retain only those terms that contribute to the energy shift at order $L^{-6}$ : 


$$
\begin{aligned}
X= & -\left(c^{2} u_{11}+2 c s u_{12}+s^{2} u_{22}\right)-a\left(c s\left(u_{22}-u_{11}\right)\right. \\
& \left.+\left(c^{2}-s^{2}\right) u_{12}\right)^{2}-a^{2}\left(c s\left(u_{22}-u_{11}\right)\right. \\
& \left.+\left(c^{2}-s^{2}\right) u_{12}\right)^{2}\left(c^{2} u_{22}-2 c s u_{12}+s^{2} u_{11}\right)+\cdots
\end{aligned}
$$

As in the case of the ground state, we solve the above equation iteratively. The explicit expression for the energy shift at this order is given by

$$
\begin{aligned}
\kappa^{2}-1 & =\frac{h_{0}}{L}\left(1+\frac{h_{1}}{L}+\frac{h_{2}}{L^{2}}+\frac{h_{3}}{L^{3}} \ln \frac{m L}{2 \pi}+\frac{h_{4}}{L^{3}}+\cdots\right), \\
h_{0} & =\frac{10 a}{\pi} .
\end{aligned}
$$

Our aim is to determine the coefficients $h_{1}, h_{2}, h_{3}, h_{4}$. Substituting the above solution into Eqs. (75) and (77) and recalling the definition of $u_{k l}$ from Eq. (73), we get

$$
u_{k l}=\frac{u_{k l}^{(0)}}{L}+\frac{u_{k l}^{(1)}}{L^{2}}+\frac{u_{k l}^{(2)}}{L^{3}} \ln \frac{m L}{2 \pi}+\frac{u_{k l}^{(3)}}{L^{3}}+\cdots,
$$

where

$u_{k l}^{(0)}=a_{k l}^{(0)}$,

$u_{k l}^{(1)}=b_{k l}^{(0)}-a_{k l}^{(1)} h_{0}-\bar{\Delta}_{k l}^{(0)}$,

$u_{k l}^{(2)}=-\bar{\Delta}_{k l}^{(1)}$,

$u_{k l}^{(3)}=-b_{k l}^{(1)} h_{0}-a_{k l}^{(1)} h_{0} h_{1}+a_{k l}^{(2)} h_{0}^{2}+c_{k l}-\bar{\Delta}_{k l}^{(2)}-\bar{\Delta}_{k l}^{(3)} h_{0}$.

Solving the quantization condition, we finally obtain:

$$
\begin{aligned}
h_{1}= & a\left(c^{2} u_{11}^{(0)}+2 c s u_{12}^{(0)}+s^{2} u_{22}^{(0)}\right), \\
h_{2}= & a\left(c^{2} u_{11}^{(1)}+2 c s u_{12}^{(1)}+s^{2} u_{22}^{(1)}\right)+a^{2}\left(c^{2}\left(u_{11}^{(0)}\right)^{2}+s^{2}\left(u_{22}^{(0)}\right)^{2}+\left(u_{12}^{(0)}\right)^{2}+2 c s u_{12}^{(0)}\left(u_{11}^{(0)}+u_{22}^{(0)}\right)\right), \\
h_{3}= & a\left(c^{2} u_{11}^{(2)}+2 c s u_{12}^{(2)}+s^{2} u_{22}^{(2)}\right), \\
h_{4}= & a\left(c^{2} u_{11}^{(3)}+2 c s u_{12}^{(3)}+s^{2} u_{22}^{(3)}\right)+2 a^{2}\left(c^{2} u_{11}^{(0)} u_{11}^{(1)}+s^{2} u_{22}^{(0)} u_{22}^{(1)}+u_{12}^{(0)} u_{12}^{(1)}+c s u_{12}^{(0)}\left(u_{11}^{(1)}+u_{22}^{(1)}\right)+c s u_{12}^{(1)}\left(u_{11}^{(0)}+u_{22}^{(0)}\right)\right) \\
& +a^{3}\left(2 c s\left(u_{12}^{(0)}\right)^{3}+\left(u_{12}^{(0)}\right)^{2}\left(\left(1+c^{2}\right) u_{11}^{(0)}+\left(1+s^{2}\right) u_{22}^{(0)}\right)+2 c s u_{12}^{(0)}\left(\left(u_{11}^{(0)}\right)^{2}+u_{11}^{(0)} u_{22}^{(0)}+\left(u_{22}^{(0)}\right)^{2}\right)\right. \\
& \left.+\left(c^{2}\left(u_{11}^{(0)}\right)^{3}+s^{2}\left(u_{22}^{(0)}\right)^{3}\right)\right) .
\end{aligned}
$$

This solves the quantization condition at order $L^{-6}$. In order to complete the solution, we should give explicit expressions for the coefficients $u_{k l}^{(\alpha)}$, entering the above expressions.

\section{Expansion of $\overline{\mathcal{A}}_{k l}$}

The coefficients of the expansion of the quantities $\overline{\mathcal{A}}_{k l},(k, l=1,2)$ can be directly read off from Eqs. (53), (54) and (57). The result is given below:

$$
\begin{gathered}
a_{11}^{(0)}=-\frac{1}{\pi} \mathcal{I}_{1}+\frac{2}{\pi}, \quad a_{11}^{(1)}=\frac{1}{\pi} \mathcal{J}_{1}+\frac{2}{\pi}, \quad a_{11}^{(2)}=-\frac{1}{\pi} \mathcal{K}_{1}+\frac{2}{\pi}, \\
b_{11}^{(0)}=2 \pi^{2} r, \quad b_{11}^{(1)}=-2 \pi^{2} r, \quad c_{11}=-8 \pi \frac{H_{0}(\Lambda)}{\Lambda^{2}} \\
a_{12}^{(0)}=a_{12}^{(1)}=a_{12}^{(2)}=b_{12}^{(0)}=b_{12}^{(1)}=0 \\
c_{12}=-8 \sqrt{6} \pi \frac{H_{0}(\Lambda)}{\Lambda^{2}}
\end{gathered}
$$

$a_{22}^{(0)}=-\frac{1}{\pi} \mathcal{I}_{2}-\frac{9}{\pi}, \quad a_{22}^{(1)}=\frac{1}{\pi} \mathcal{J}_{2}+\frac{17}{2 \pi}, \quad a_{22}^{(2)}=-\frac{1}{\pi} \mathcal{K}_{2}-\frac{33}{4 \pi}$,

$b_{22}^{(0)}=\frac{1}{2} \pi^{2} r, \quad b_{22}^{(1)}=-2 \pi^{2} r, \quad c_{22}=-48 \pi \frac{H_{0}(\Lambda)}{\Lambda^{2}}$.

\section{Expansion of $\overline{\boldsymbol{\Delta}}_{\boldsymbol{k} l}$}

In order to obtain the expansion of $\bar{\Delta}_{k l}$, we have to consider the quantity

$\Delta(\mathbf{p}, \mathbf{q} ; E)=\frac{1}{L^{3}} \sum_{\mathbf{k}}^{\prime} Z(\mathbf{p}, \mathbf{k} ; E) \tau_{L}(\mathbf{k} ; E) Z(\mathbf{k}, \mathbf{q} ; E)+\cdots$,

where $\mathbf{p}=\frac{2 \pi}{L} \mathbf{i}$ and $\mathbf{q}=\frac{2 \pi}{L} \mathbf{j}$ belong either to shell $s=1$ or $s=2$, and the prime over the summation indicates that $\mathbf{k}^{2}=\left(\frac{2 \pi}{L}\right)^{2} \mathbf{n}^{2}>\left(\frac{2 \pi}{L}\right)^{2}$ (the vector $\mathbf{k}$ belongs to the shell $r>2)$.

The expansion of the above quantity in the limit of the large $L$ proceeds in the same way as for the ground state: 


$$
L^{-3} \Delta(\mathbf{p}, \mathbf{q} ; E)=\frac{\Delta^{(0)}(\mathbf{p}, \mathbf{q})}{L^{2}}+\frac{\Delta^{(1)}(\mathbf{p}, \mathbf{q})}{L^{3}} \ln \frac{m L}{2 \pi}+\frac{\Delta^{(2)}(\mathbf{p}, \mathbf{q})}{L^{3}}+\left(\kappa^{2}-1\right) \frac{\Delta^{(3)}(\mathbf{p}, \mathbf{q})}{L^{2}}+O\left(L^{-4}\right),
$$

where

$$
\begin{aligned}
\Delta^{(0)}(\mathbf{p}, \mathbf{q}) & =-\frac{a \mathcal{J}(\mathbf{i}, \mathbf{j})}{2 \pi^{3}}, & \Delta^{(1)}(\mathbf{p}, \mathbf{q}) & =-\frac{2 \sqrt{3} a^{2}}{\pi}+\frac{8 a^{2}}{3}, \\
\frac{H_{0}(\Lambda)}{\Lambda^{2}}+\Delta^{(2)}(\mathbf{p}, \mathbf{q}) & =\hat{\mathcal{M}}+\frac{a^{2}}{(2 \pi)^{3}} \mathcal{B}(\mathbf{i}, \mathbf{j}), & \Delta^{(3)}(\mathbf{p}, \mathbf{q}) & =-\frac{a}{\pi^{3}} \mathcal{K}(\mathbf{i}, \mathbf{j}),
\end{aligned}
$$

the integer vectors $\mathbf{i}, \mathbf{j}$ are given by $\mathbf{i}=\frac{L \mathbf{p}}{2 \pi}, \mathbf{j}=\frac{L \mathbf{q}}{2 \pi}$, and

$$
\begin{aligned}
& \mathcal{J}(\mathbf{i}, \mathbf{j})=\sum_{|\mathbf{n}| \neq 0,1}^{\infty} \frac{1}{\mathbf{i}^{2}+\mathbf{i n}+\mathbf{n}^{2}-1} \frac{1}{\mathbf{n}^{2}+\mathbf{n} \mathbf{j}+\mathbf{j}^{2}-1}, \\
& \mathcal{K}(\mathbf{i}, \mathbf{j})=\frac{1}{2} \sum_{|\mathbf{n}| \neq 0,1}^{\infty}\left(\frac{1}{\left(\mathbf{i}^{2}+\mathbf{i n}+\mathbf{n}^{2}-1\right)^{2}} \frac{1}{\mathbf{n}^{2}+\mathbf{n} \mathbf{j}+\mathbf{j}^{2}-1}+\frac{1}{\mathbf{i}^{2}+\mathbf{i n}+\mathbf{n}^{2}-1} \frac{1}{\left(\mathbf{n}^{2}+\mathbf{n} \mathbf{j}+\mathbf{j}^{2}-1\right)^{2}}\right), \\
& \mathcal{B}(\mathbf{i}, \mathbf{j})=-16 \pi^{2} \sqrt{3}\left(\frac{1}{2}(1-\ln 3)+\frac{\sqrt{3} \pi}{18}\right)-4 \pi \sqrt{3} \mathcal{L}(\mathbf{i}, \mathbf{j})+\frac{4}{\pi} \mathcal{G}(\mathbf{i}, \mathbf{j})+\frac{8}{\pi}\left(2 \mathcal{Q}(\mathbf{i}, \mathbf{j})-\mathcal{I}_{0}\right) . \\
& \mathcal{L}(\mathbf{i}, \mathbf{j})=\lim _{N \rightarrow \infty}\left(\sum_{|\mathbf{n}| \neq 0,1}^{N} \frac{\sqrt{\frac{3}{4} \mathbf{n}^{2}-1}}{\left(\mathbf{i}^{2}+\mathbf{i n}+\mathbf{n}^{2}-1\right)\left(\mathbf{n}^{2}+\mathbf{n} \mathbf{j}+\mathbf{j}^{2}-1\right)}-4 \pi \ln N\right), \\
& \mathcal{Q}(\mathbf{i}, \mathbf{j})=\sum_{N \rightarrow \mathbf{n} \mid \neq 0,1}^{\infty} \frac{\mathcal{I}(\mathbf{n})}{\left(\mathbf{i}^{2}+\mathbf{i n}+\mathbf{n}^{2}-1\right)\left(\mathbf{n}^{2}+\mathbf{n} \mathbf{j}+\mathbf{j}^{2}-1\right)}\left(\frac{1}{2} \sum_{|\mathbf{n}| \neq 0,1}^{N} \sum_{|\mathbf{m}| \neq 0,1}^{N} \frac{1}{\mathbf{i}^{2}+\mathbf{i n}+\mathbf{n}^{2}-1} \frac{1}{\mathbf{n}^{2}+\mathbf{n m}+\mathbf{m}^{2}-1} \frac{1}{\mathbf{m}^{2}+\mathbf{m} \mathbf{j}+\mathbf{j}^{2}-1}-\frac{4 \pi^{4}}{3} \ln N\right) .
\end{aligned}
$$

Note that the quantities, defined in the infinite volume (the threshold amplitude $\hat{\mathcal{M}}$, the coefficient in front of the logarithm and the integral $\mathcal{I}_{0}$ ) are the same as in the case of the ground-state shift.

Recalling now the definition

$$
\bar{\Delta}_{k l}=\frac{8 \pi \sqrt{\theta(k) \theta(l)}}{G L^{3}} \sum_{g \in O_{h}} \Delta(g \mathbf{p}, \mathbf{q}, E),
$$

we get:

$$
\begin{aligned}
\bar{\Delta}_{k l}^{(0)} & =-\frac{4 a}{\pi^{2}} \mathcal{J}_{k l}, \\
\bar{\Delta}_{k l}^{(1)} & =\left(-16 \sqrt{3}+\frac{64 \pi}{3}\right) a^{2} \sqrt{\theta(k) \theta(l)}, \\
\bar{\Delta}_{k l}^{(2)}-c_{k l} & =8 \pi \hat{\mathcal{M}} \sqrt{\theta(k) \theta(l)}+\frac{a^{2}}{\pi^{2}} \mathcal{B}_{k l}, \\
\bar{\Delta}_{k l}^{(3)} & =-\frac{8 a}{\pi^{2}} \mathcal{K}_{k l},
\end{aligned}
$$

where

$$
\begin{aligned}
\mathcal{B}_{k l}= & {\left[-16 \pi^{2} \sqrt{3}\left(\frac{1}{2}(1-\ln 3)+\frac{\sqrt{3} \pi}{18}\right)-\frac{8}{\pi} \mathcal{I}_{0}\right] \sqrt{\theta(k) \theta(l)} } \\
& -4 \pi \sqrt{3} \mathcal{L}_{k l}+\frac{4}{\pi} \mathcal{G}_{k l}+\frac{16}{\pi} \mathcal{Q}_{k l} .
\end{aligned}
$$

In the above equations, $\theta(1)=1, \theta(2)=6$, and the quantities $\mathcal{J}_{k l}, \quad \mathcal{K}_{k l}, \quad \mathcal{L}_{k l}, \quad \mathcal{G}_{k l}, \quad \mathcal{Q}_{k l}$ are defined in Appendix C.

Our derivation of the energy shift of the first excited level in the irrep $A_{1}^{+}$is now complete-all quantities, contributing to $h_{1}, h_{2}, h_{3}, h_{4}$ from Eq. (81) are explicitly defined. Next, we shall demonstrate, how an analogous formula can be obtained in the irrep $E^{+}$.

\section{E. The quantization condition in the irrep $E^{+}$}

The irrep $E^{+}$is two-dimensional. Thus, in the quantization condition (51), the labels $\sigma, \rho$ now run from 1 to 2 . Let us start from shell 1 . Projecting ${ }^{6}$ the driving term $Z$ onto the irrep $E^{+}$, we obtain that, for an arbitrary $s$,

\footnotetext{
${ }^{6}$ The matrices of different irreps of the octahedral group and the little groups thereof can be found in Refs. [37-39].
} 


$$
Z_{\sigma \rho}^{E^{+}}(1, s)=Z_{\sigma \rho}^{E^{+}}(s, 1)=0 .
$$

At a first glance, this leads to the controversy since, as seen from Eq. (51), one of the roots decouples from the rest and obeys the equation

$$
\tau_{L}^{-1}(s=1, E)=0 .
$$

This root would correspond to the pure two-body level, without an interaction with the spectator. It is, however, easy to show that we are dealing here with a spurious solution. To this end, one has to return one step back and consider the system of linear equations, which yield the quantization condition, prior to projecting onto the different irreps. Taking into account Eq. (5), this system is written down as

$$
\tau_{L}^{-1}(\mathbf{p} ; E) f(\mathbf{p})-\frac{1}{L^{3}} \sum_{\mathbf{q}} Z(\mathbf{p}, \mathbf{q} ; E) f(\mathbf{q})=0,
$$

see also Ref. [20]. Here, $f(\mathbf{p})$ is a solution. This system has nontrivial solutions, if and only if the determinant vanishes, that leads to the quantization condition already considered above.

Projecting now this system of equations onto the irrep $E^{+}$, it is seen that the projection of the vector $f(\mathbf{p})$ on the first shell, corresponding to $\mathbf{p}=0$, vanishes identically due to symmetry reasons:

$$
\sum_{g \in O_{h}}\left(T_{\sigma \rho}^{E^{+}}(g)\right)^{*} f(\mathbf{0})=0,
$$

and, hence, the first two lines $s=1, \sigma=1,2$ of the projected equations, which correspond to the projection onto the first shell, turn into a trivial identity $0=0$ and should be deleted [we remind the reader that the projection of the driving term $Z$ onto the first shell also vanishes, see Eq. (92)]. Thus, the spurious solution disappears. This simple example shows a pattern, suggesting how to avoid the spurious solutions in general: if a projection $f_{\rho \sigma}^{\Gamma}(s)$ of a generic vector $f(\mathbf{p})$ identically vanishes for a given $s$ and $\rho$ (the second index $\sigma$ is fixed, see Ref. [20] for details), one has to delete the corresponding rows/columns in the quantization condition from the beginning. Note also that deleting the entries corresponding to the first shell, makes the solution of the quantization condition easier-unlike the case with the irrep $A_{1}^{+}$, now there is no need for the special treatment of the singular terms in the matrix $\mathcal{A}$.

Now, let us turn to the shell $s=2$, corresponding to $|\mathbf{p}|=2 \pi / L$ and choose the reference momentum as $\mathbf{p}_{0}=2 \pi / L(0,0,1)$. In total, there are six different momenta in the second shell, which are obtained from the reference momentum by acting the group elements belonging to the set $\mathcal{S}=\left\{g_{1}=\mathbb{I}, g_{2}, g_{3}, I, I g_{2}, I g_{3}\right\}$ (II is the identity matrix and $I$ denotes the inversion of all components of the vector; the numeration is the same as, e.g., in Table B.1 of Ref. [39]). Namely, $g_{2} \mathbf{p}_{0}=\mathbf{p}_{0}^{\prime}=$ $2 \pi / L(0,1,0)$ and $g_{3} \mathbf{p}_{0}=\mathbf{p}_{0}^{\prime \prime}=2 \pi / L(1,0,0)$. Further, let $\hat{g}$ denote the elements of a subgroup in $O_{h}$ that leave the reference vector $\mathbf{p}_{0}$ invariant. This subgroup $\hat{\mathcal{G}}$ consists of the following elements: $\hat{g}=g_{1}, g_{14}, g_{15}, g_{24}, I g_{18}, I g_{19}$, $I g_{22}, I g_{23}$. Consequently,

$$
\begin{aligned}
Z_{\sigma \rho}^{E^{+}}(2, s) & =\sum_{g^{\prime} \in \mathcal{S}} \sum_{\hat{g} \in \hat{\mathcal{G}}}\left(T_{\rho \sigma}^{E^{+}}\left(g^{\prime} \hat{g}\right)\right)^{*} Z\left(g^{\prime} \hat{g} \mathbf{p}_{0}, \mathbf{q}_{0} ; E\right) \\
& =\sum_{g^{\prime} \in \mathcal{S}}\left(T_{\rho \lambda}^{E^{+}}\left(g^{\prime}\right)\right)^{*} Z\left(g^{\prime} \mathbf{p}_{0}, \mathbf{q}_{0} ; E\right)\left\{\sum_{\hat{g} \in \hat{\mathcal{G}}}\left(T_{\lambda \sigma}^{E^{+}}(\hat{g})\right)^{*}\right\} \\
& =\left(\begin{array}{ll}
a(s) & 0 \\
b(s) & 0
\end{array}\right)_{\rho \sigma},
\end{aligned}
$$

where $\mathbf{q}_{0}$ denotes the reference momentum for the shell $s$ and

$$
\begin{aligned}
a(s)= & 8\left(Z\left(\mathbf{p}_{0}, \mathbf{q}_{0} ; E\right)+Z\left(-\mathbf{p}_{0}, \mathbf{q}_{0} ; E\right)-\frac{1}{2} Z\left(\mathbf{p}_{0}^{\prime}, \mathbf{q}_{0} ; E\right)\right. \\
& -\frac{1}{2} Z\left(-\mathbf{p}_{0}^{\prime}, \mathbf{q}_{0} ; E\right)-\frac{1}{2} Z\left(\mathbf{p}_{0}^{\prime \prime}, \mathbf{q}_{0} ; E\right) \\
& \left.-\frac{1}{2} Z\left(-\mathbf{p}_{0}^{\prime \prime}, \mathbf{q}_{0} ; E\right)\right), \\
b(s)= & -4 \sqrt{3}\left(Z\left(\mathbf{p}_{0}^{\prime}, \mathbf{q}_{0} ; E\right)+Z\left(-\mathbf{p}_{0}^{\prime}, \mathbf{q}_{0} ; E\right)\right. \\
& \left.-Z\left(\mathbf{p}_{0}^{\prime \prime}, \mathbf{q}_{0} ; E\right)-Z\left(-\mathbf{p}_{0}^{\prime \prime}, \mathbf{q}_{0} ; E\right)\right) .
\end{aligned}
$$

In particular, $b(s)=0$ for the shell $s=2$.

Now, it is straightforward to convince oneself that the nondiagonal matrix elements of the matrix $\mathcal{A}_{2 s}^{2 \sigma}$ and $\mathcal{A}_{s 2}^{\sigma 2}$ vanish for $\sigma=1,2$. Using the same method as for the shell $s=1$, it can be shown that this row/column in the matrix $\mathcal{A}$ should be deleted, in order to avoid spurious solutions. Consequently, the quantization condition is rewritten in the following form ${ }^{7}$

$$
\mathcal{A}_{22}^{11}=\Delta_{22}^{11},
$$

where

$$
\Delta_{22}^{11}=\frac{48 \pi}{G L^{3}} \sum_{g \in O_{h}}\left(T_{11}^{E^{+}}(g)\right)^{*} \Delta\left(g \mathbf{p}_{0}, \mathbf{p}_{0} ; E\right)
$$

where $\mathbf{p}_{0}$ is the reference momentum for the shell $s=2$ and the quantity $\Delta(\mathbf{p}, \mathbf{p} ; E)$ is defined in Eq. (85). Further,

\footnotetext{
${ }^{7}$ In the matrix $\mathcal{A}_{r s}^{\sigma \rho}$ the lower indices, as before, label the shells, whereas the upper indices $\sigma, \rho=1,2$ are used to label the basis vectors of the irrep $E^{+}$. In order to avoid clutter of various indices, we further omit the label $E^{+}$from all expressions.
} 


$$
\mathcal{A}_{22}^{11}=-\frac{1}{a}-\frac{4}{\pi L} \frac{1}{1-\kappa^{2}}+\overline{\mathcal{A}}_{22}^{11}
$$

the expansion of the quantity $\overline{\mathcal{A}}_{22}^{11}$ is similar but not identical to Eq. (76) (note the absence of the term with $c_{22}$ ):

$$
\begin{aligned}
\overline{\mathcal{A}}_{22}^{11}= & \left(\frac{a_{22}^{(0) \prime}}{L}+\frac{b_{22}^{(0) \prime}}{L^{2}}\right)+\left(\frac{a_{22}^{(1) \prime}}{L}+\frac{b_{22}^{(1) \prime}}{L^{2}}\right)\left(1-\kappa^{2}\right) \\
& +\frac{a_{22}^{(2) \prime}}{L}\left(1-\kappa^{2}\right)^{2}+\cdots,
\end{aligned}
$$

where

$a_{22}^{(0) \prime}=-\frac{1}{\pi} \mathcal{I}_{2}+\frac{3}{\pi}, \quad a_{22}^{(1) \prime}=\frac{1}{\pi} \mathcal{J}_{2}-\frac{7}{2 \pi}, \quad a_{22}^{(2) \prime}=-\frac{1}{\pi} \mathcal{K}_{2}+\frac{15}{4 \pi}$,

$b_{22}^{(0) \prime}=\frac{1}{2} \pi^{2} r, \quad b_{22}^{(1) \prime}=-2 \pi^{2} r$.

Further, the expansion of the quantity $\Delta_{22}^{11}$ is given by, cf. with Eq. (75):

$$
\Delta_{22}^{11}=\frac{\Delta_{22}^{(0) \prime}}{L^{2}}+\frac{\Delta_{22}^{(2) \prime}}{L^{3}}+\frac{\Delta_{22}^{(3) \prime}}{L^{2}}\left(\kappa^{2}-1\right)+\cdots
$$

where

$$
\begin{aligned}
& \Delta_{22}^{(0) \prime}=-\frac{4 a}{\pi^{2}} \mathcal{J}_{22}^{\prime}, \\
& \Delta_{22}^{(2) \prime}=\frac{a^{2}}{\pi^{2}}\left\{-4 \pi \sqrt{3} \mathcal{L}_{22}^{\prime}+\frac{4}{\pi} \mathcal{G}_{22}^{\prime}+\frac{16}{\pi} \mathcal{Q}_{22}^{\prime}\right\}, \\
& \Delta_{22}^{(3) \prime}=-\frac{8 a}{\pi^{2}} \mathcal{K}_{22}^{\prime} .
\end{aligned}
$$

Note again the absence of the logarithmic term and the threshold amplitude, as compared to the case of the irrep $A_{1}^{+}$. The numerical constants in the above equation are given in Appendix C.

Finally, the expansion of the energy shift is given by

$$
\kappa^{2}-1=\frac{h_{0}^{\prime}}{L}\left(1+\frac{h_{1}^{\prime}}{L}+\frac{h_{2}^{\prime}}{L^{2}}+\frac{h_{4}^{\prime}}{L^{3}}\right)
$$

where

$$
\begin{aligned}
h_{0}^{\prime} & =\frac{4 a}{\pi}, \\
h_{1}^{\prime} & =a u_{22}^{(0) \prime}, \\
h_{2}^{\prime} & =\left(a u_{22}^{(0) \prime}\right)^{2}+a u_{22}^{(1) \prime}, \\
h_{4}^{\prime} & =\left(a u_{22}^{(0) \prime}\right)^{3}+2 a^{2} a_{22}^{(0) \prime} u_{22}^{(1) \prime}+a u_{22}^{(3) \prime},
\end{aligned}
$$

and

$$
\begin{aligned}
& u_{22}^{(0) \prime}=a_{22}^{(0) \prime}, \\
& u_{22}^{(1) \prime}=b_{22}^{(0) \prime}-a_{22}^{(1) \prime} h_{0}^{\prime}-\Delta_{22}^{(0) \prime}, \\
& u_{22}^{(3) \prime}=-b_{22}^{(1) \prime} h_{0}^{\prime}-a_{22}^{(1) \prime} h_{0}^{\prime} h_{1}^{\prime}+a_{22}^{(2) \prime}\left(h_{0}^{\prime}\right)^{2}-\Delta_{22}^{(2) \prime}-\Delta_{22}^{(3) \prime} h_{0}^{\prime} .
\end{aligned}
$$

This is the final expression for the energy shift at order $L^{-6}$. As one sees, at this order it contains neither the threshold amplitude, nor the logarithmic term. The reason for this is the symmetry: both terms do not depend on the direction. Averaging them over the octahedral group yields zero in all irreps except $A_{1}^{+}$. A particle-dimer interaction in higher partial waves would potentially contribute here but, according to the power counting, this contribution comes at higher order in $1 / L$.

\section{COMPARISON TO SOME EARLIER WORK}

\section{A. Comparison with Hansen and Sharpe}

First of all, it should be pointed out that the framework, used in the present paper, is nonrelativistic. Therefore, the relativistic corrections, which are present in the expression from Refs. $[8,9,16]$ at order $L^{-6}$, cannot be reproduced. The work on the relativistic version of our approach is in progress, and the results will be reported elsewhere.

Further, in order to carry out the comparison, we have to relate our threshold amplitude $\hat{\mathcal{M}}$ with the amplitude $M_{3, t h r}$, which appears in the above papers. We remind the reader that our threshold amplitude $\hat{\mathcal{M}}$ is defined as follows: consider the particle-dimer scattering amplitude $\mathcal{M}(\mathbf{p}, \mathbf{q} ; E)$ at $\mathbf{p}=\mathbf{q}=0$ and $-m E=\varepsilon^{2} \rightarrow 0$. This amplitude can be written in the following form:

$$
\mathcal{M}=I_{0}+I_{1}+I_{2}+\overline{\mathcal{M}}
$$

where $I_{0}$ is the tree-level exchange term and $I_{1}, I_{2}$ denote the first and the second iteration of $I_{0}$. All the rest, which is denoted by $\overline{\mathcal{M}}$, is regular, as $\varepsilon \rightarrow 0$, whereas $I_{0}, I_{1}, I_{2}$ contain the singularities $\varepsilon^{-2}, \varepsilon^{-1}$ and $\ln \frac{\varepsilon}{m}$. It can be shown that

$$
\begin{aligned}
I_{0}+I_{1}+I_{2}= & \frac{4 a}{\pi \Lambda}-\frac{2 a^{2}}{\pi} \ln \frac{\Lambda}{m}\left(\sqrt{3}-\frac{4 \pi}{3}\right)-\frac{a^{2}}{(2 \pi)^{3}} \delta \\
& + \text { singular terms in } \varepsilon,
\end{aligned}
$$

where $\delta$ is given in Eq. (42). The threshold amplitude $\hat{\mathcal{M}}$ is obtained from $\mathcal{M}$ by deleting these singularities. Hence,

$$
\hat{\mathcal{M}}=\frac{4 a}{\pi \Lambda}-\frac{2 a^{2}}{\pi} \ln \frac{\Lambda}{m}\left(\sqrt{3}-\frac{4 \pi}{3}\right)-\frac{a^{2}}{(2 \pi)^{3}} \delta+\overline{\mathcal{M}} .
$$


On the other hand, following Refs. [8,9,16], one may introduce a different splitting of the same amplitude:

$$
\mathcal{M}=I_{0}^{H}+I_{1}^{H}+I_{2}^{H}+\overline{\mathcal{M}}^{H}
$$

where the superscript $H$ indicates that the ultraviolet regularization, instead of the sharp cutoff $\Lambda$, is carried out by using the smooth cutoff function $H$, introduced in these papers. The prescription, used in Refs. $[8,9,16]$, consists in dropping the singular pieces $I_{0}^{H}, I_{1}^{H}, I_{2}^{H}$ altogether. The particle-dimer threshold amplitude within this prescription is equal merely to $\overline{\mathcal{M}}^{H}$. The three particle threshold amplitude $\mathcal{M}_{3}$ is, modulo overall normalization, which will be considered later, proportional to $\overline{\mathcal{M}}^{H}$.

From these definitions, it is straightforward to obtain a linear relation between $\hat{\mathcal{M}}$ and $\overline{\mathcal{M}}^{H}$ :

$$
\overline{\mathcal{M}}^{H}=\left(\hat{\mathcal{M}}+\frac{a^{2}}{(2 \pi)^{3}} \delta\right)+\Delta S^{\prime}+\Delta S^{\prime \prime}+\Delta S^{\prime \prime \prime},
$$

where

$$
\begin{aligned}
\Delta S^{\prime}= & -8 \pi a \int^{\Lambda} \frac{d^{3} \mathbf{k}}{(2 \pi)^{3}} \frac{1}{\mathbf{k}^{4}}-\frac{4 a}{\pi \Lambda}+8 \pi a \int \frac{d^{3} \mathbf{k}}{(2 \pi)^{3}} \frac{H^{2}\left(\frac{k}{m}\right)}{\mathbf{k}^{4}}, \\
\Delta S^{\prime \prime}= & -4 \sqrt{3} \pi a^{2} \int^{\Lambda} \frac{d^{3} \mathbf{k}}{(2 \pi)^{3}} \frac{1}{|\mathbf{k}|^{3}}+\frac{2 a^{2} \sqrt{3}}{\pi} \ln \frac{\Lambda}{m} \\
& +4 \sqrt{3} \pi a^{2} \int \frac{d^{3} \mathbf{k}}{(2 \pi)^{3}} \frac{H^{3}\left(\frac{k}{m}\right)}{|\mathbf{k}|^{3}}, \\
\Delta S^{\prime \prime \prime}= & 64 \pi^{2} a^{2} \int^{\Lambda} \frac{d^{3} \mathbf{k}}{(2 \pi)^{3}} \int^{\Lambda} \frac{d^{3} \mathbf{q}}{(2 \pi)^{3}} \frac{1}{\mathbf{k}^{2}\left(\mathbf{k}^{2}+\mathbf{k q}+\mathbf{q}^{2}\right) \mathbf{q}^{2}} \\
& -\frac{8 a^{2}}{3} \ln \frac{\Lambda}{m} \\
& -64 \pi^{2} a^{2} \int \frac{d^{3} \mathbf{k}}{(2 \pi)^{3}} \int \frac{d^{3} \mathbf{g}}{(2 \pi)^{3}} \frac{H^{2}\left(\frac{k}{m}\right) H^{2}\left(\frac{q}{m}\right)}{\mathbf{k}^{2}\left(\mathbf{k}^{2}+\mathbf{k q}+\mathbf{q}^{2}\right) \mathbf{q}^{2}} .
\end{aligned}
$$

Note that, in the above integrals, there is no need for infrared regularization anymore.

In order to complete the comparison, one has next to relate various infinite sums over integer numbers, which appear at order $L^{-6}$ in Refs. $[8,9,16]$ and in the present paper. In the above papers, the ultraviolet regularization is again carried out by introducing the smooth cutoff function $H$. Thus, the difference emerges only at high momenta due to the different ultraviolet cutoff and, consequently, one may safely perform the limit $L \rightarrow \infty$ in the differences, replacing the summation by integration. As a result, one obtains:

$$
\begin{aligned}
\mathcal{C}_{F} & =-4 \mathcal{G}, \\
\Delta S^{\prime} & =\frac{8 \pi a}{m} \mathcal{C}_{3}, \\
\Delta S^{\prime \prime} & =\frac{4 \pi a^{2} \sqrt{3}}{(2 \pi)^{3}}\left(\frac{1}{4 \pi^{2} \sqrt{3}} \mathcal{C}_{4}-\mathcal{L}\right), \\
\Delta S^{\prime \prime \prime} & =\frac{a^{2}}{8 \pi^{4}} \mathcal{C}_{5}+\frac{2 a^{2}}{\pi^{4}} \mathcal{Q},
\end{aligned}
$$

where $\mathcal{C}_{F}, \mathcal{C}_{3}, \mathcal{C}_{4}, \mathcal{C}_{5}$ are the numerical constants, appearing in Refs. $[8,9,16]$. Taking into account Eqs. (113) and (114), it is seen that all $H$-dependent terms in the energy shift expression from Refs. [8,9,16] cancel (as they indeed should), and this expression algebraically coincides with our expression up to the replacement $\hat{\mathcal{M}}+\frac{a^{2}}{(2 \pi)^{3}} \delta \rightarrow \overline{\mathcal{M}}^{H}$.

What remains to be fixed is the normalization coefficient in the relation of $\overline{\mathcal{M}}^{H}$ and $M_{3, \mathrm{thr}}$. Most easily this can be done by comparing the normalization of the tree-level exchange term $I_{0}$ in the present paper and in Refs. $[8,9,16]$. The result reads

$$
9 a^{2} \overline{\mathcal{M}}^{H}=\frac{1}{64 \pi^{2}(2 m)^{2}} M_{3, \mathrm{thr}}
$$

where the combinatorial factor 9 emerges, when one relates the particle-dimer scattering amplitude to the three-particle amplitude, with all permutations of particles in the initial and final states. Using this relation, it is then seen that the energy shift from Refs. $[8,9,16]$ exactly reproduces our result.

\section{B. Comparison with Beane, Detmold and Savage}

In order to compare our result with Ref. [34], a perturbative expansion of the quantity $\hat{\mathcal{M}}$ should be carried out. To this end, one has to relate the particle-dimer coupling $\frac{H_{0}(\Lambda)}{\Lambda^{2}}$, which emerges in our expressions, to the three-body coupling constant $\eta_{3}^{r}(\mu)$ from Ref. [34], where the ultraviolet divergence is removed by using dimensional regularization, and $\mu$ is the scale of the dimensional regularization. If there are no derivative two-body couplings, that lead to the nonzero effective range, these two couplings at tree level are merely proportional to each other. However, if the derivative terms are present, the relation is more complicated, and can be figured out in the following manner. Consider the particle-dimer Lagrangian ${ }^{8}$

\footnotetext{
${ }^{8}$ Here, for convenience, we use a dimer formulation that is different from one described in Ref. [18]. Namely, here the effective-range term is included in the kinetic term of the dimer and not in the derivative particle-dimer vertex. Both formulations are equivalent, leading to the same $S$-matrix.
} 


$$
\begin{aligned}
\mathcal{L}= & \psi^{\dagger}\left(i \partial_{0}-\frac{\nabla^{2}}{2 m}\right) \psi+d^{\dagger}\left(i \partial_{0}+\frac{\nabla^{2}}{4 m}+\Delta\right) d \\
& -\frac{g}{\sqrt{2}}\left(d^{\dagger} \psi \psi+\text { H.c. }\right)+h d^{\dagger} d \psi^{\dagger} \psi+\cdots,
\end{aligned}
$$

where $\psi$ and $d$ stand for the nonrelativistic particle and dimer fields, respectively. The quantity $h$ is proportional to the particle-dimer coupling $h=2 m g^{2} \frac{H_{0}(\Lambda)}{\Lambda^{2}}$.

Let us now integrate out the dimer field $d$ that leads to the three-particle Lagrangian. At tree level, one may set the emerging determinant to unity. Expanding the obtained Lagrangian in powers of $\frac{1}{\Delta}$, we get

$$
\begin{aligned}
\mathcal{L}= & \psi^{\dagger}\left(i \partial_{0}-\frac{\nabla^{2}}{2 m}\right) \psi-\frac{g^{2}}{2 \Delta} \psi^{\dagger} \psi^{\dagger} \\
& \times\left\{1-\frac{1}{\Delta}\left(i \partial_{0}+\frac{\nabla^{2}}{4 m}-h \psi^{\dagger} \psi\right)+\cdots\right\} \psi \psi
\end{aligned}
$$

Using the equations of motion

$$
\left(i \partial_{0}+\frac{\nabla^{2}}{2 m}-\frac{g^{2}}{\Delta} \psi^{\dagger} \psi\right) \psi=0
$$

in the last term, one can rewrite the Lagrangian in the following form

$$
\begin{aligned}
\mathcal{L}= & \psi^{\dagger}\left(i \partial_{0}-\frac{\nabla^{2}}{2 m}\right) \psi-\frac{g^{2}}{2 \Delta}\left(\psi^{\dagger} \psi\right)^{2} \\
& -\frac{g^{2}}{4 m \Delta^{2}}\left(\psi^{\dagger} \psi^{\dagger} \psi \nabla^{2} \psi+\text { H.c. }\right) \\
& +\left(\frac{g^{2} h}{2 \Delta^{2}}+\frac{g^{4}}{\Delta^{3}}\right)\left(\psi^{\dagger} \psi\right)^{3}+\frac{g^{2}}{8 m \Delta^{2}} \psi^{\dagger} \psi^{\dagger} \nabla^{2}(\psi \psi)+\cdots
\end{aligned}
$$

From this, one may read off the matching conditions of the couplings:

$$
\begin{aligned}
& \frac{g^{2}}{\Delta}=\frac{4 \pi a}{m}, \quad \frac{1}{m \Delta}=-\frac{1}{2} a r, \\
& \eta_{3}=-6\left(\frac{g^{2} h}{2 \Delta^{2}}+\frac{g^{4}}{\Delta^{3}}\right)=-m\left(\frac{4 \pi a}{m}\right)^{2}\left(\frac{H_{0}(\Lambda)}{\Lambda^{2}}-\frac{1}{2} a r\right) .
\end{aligned}
$$

Here, $\eta_{3}$ is the six-particle coupling from Ref. [34].

Now, we are in a position to discuss a seeming disagreement between our result (which, as mentioned, agrees with Refs. [8,9,16]) and the result of Ref. [34]. Namely, the effective-range term equals to $\frac{6 \pi a^{2} r}{L^{3}}$ in our approach vs $\frac{2 \pi a^{2} r}{L^{3}}$ in Ref. [34]. The solution of the puzzle is as follows: it is shown that the matching of the couplings $\frac{H_{0}(\Lambda)}{\Lambda^{2}}$ and $\eta_{3}$ at tree level contains an additive term $-\frac{1}{2} a r$ with a fixed coefficient, which exactly cancels this difference and makes the results consistent. ${ }^{9}$

The calculation of the three-body energy shift has been carried out in Ref. [33] as well by using a different technique. Here, we do not carry out a detailed comparison with Ref. [33], limiting ourselves to a brief comment. As the author of Ref. [33] suggests, the results of Refs. [34,33] are identical, if the three-body couplings in both approaches are matched appropriately. In particular, the matching condition involves an additive contribution, proportional to the effective range $r$ (see, e.g., Eq. (115) of Ref. [33]). We would like to stress here again that the coefficient of the effective-range term at lowest order is fixed. Consequently, in order to demonstrate the equivalence of the two approaches, it still remains to be shown that one gets the right coefficient in the matching condition-in analogy to the discussion above.

In order to complete the comparison of our result with Ref. [34], we still have to calculate the singular pieces of the threshold amplitude in the dimensional regularization which is used in Ref. [34] (we are using the cutoff regularization throughout this paper). We start with the contribution to the particle-dimer amplitude, which corresponds to one iteration of the driving term $Z$ (an infinitevolume analog of $S_{1}$ considered in Sec. III B). Only the infrared-singular piece is of interest here (the rest is not shown explicitly). Giving the energy a negative infinitesimal shift $-m E=\varepsilon^{2}$ and writing down the dimer propagator as a sum of $0,1,2 \ldots$ bubbles, this particular contribution is given by

$$
\begin{aligned}
S_{1}^{d}= & -8 \pi a \int \frac{d^{d} \mathbf{k}}{(2 \pi)^{d}} \frac{1}{\left(\mathbf{k}^{2}+\varepsilon^{2}\right)^{2}}+\frac{1}{2}(8 \pi a)^{2} \int \frac{d^{d} \mathbf{k}}{(2 \pi)^{d}} \\
& \times \int \frac{d^{d} \mathbf{q}}{(2 \pi)^{d}} \frac{1}{\left(\mathbf{k}^{2}+\varepsilon^{2}\right)^{2}\left(\mathbf{k}^{2}+\mathbf{k} \mathbf{q}+\mathbf{q}^{2}+\varepsilon^{2}\right)}+\cdots,
\end{aligned}
$$

where the ellipses stand for more bubbles (all of them are regular at threshold and are thus omitted here) and $d$ denotes the dimension of space. Carrying out calculations, up to the terms that vanish at $\varepsilon \rightarrow 0$, one obtains:

$$
\begin{aligned}
S_{1}^{d}= & \frac{2 a}{\varepsilon}-\frac{\sqrt{3}\left(\mu^{2}\right)^{d-3} a^{2}}{\pi}\left[\frac{1}{3-d}+\Gamma^{\prime}(1)+\ln 4 \pi-\ln \frac{\varepsilon^{2}}{\mu^{2}}\right. \\
& \left.+1-\ln 2+\frac{1}{2} \ln 3-\frac{\sqrt{3} \pi}{9}\right] .
\end{aligned}
$$

\footnotetext{
${ }^{9}$ Note that such an effective range-dependent shift in the threebody coupling $H_{0}$ also appears in the infinite volume when the leading effective range correction is included [40,41]. The coefficient of this shift contains no free parameter and is fully determined by leading-order information.
} 
On the other hand, carrying out calculations in the cutoff regularization, one obtains:

$$
\begin{aligned}
S_{1}^{\Lambda} & =-8 \pi a^{2} \int^{\Lambda} \frac{d^{3} \mathbf{k}}{(2 \pi)^{3}} \frac{1}{\left(\mathbf{k}^{2}+\varepsilon^{2}\right)^{2}} \sqrt{\frac{3}{4} \mathbf{k}^{2}+\varepsilon^{2}} \\
& =\frac{2 a}{\varepsilon}-\frac{\sqrt{3} a^{2}}{\pi}\left[-\ln \frac{\varepsilon^{2}}{\Lambda^{2}}+\ln 3-\frac{\sqrt{3} \pi}{9}-1\right] .
\end{aligned}
$$

Subtracting the above two equations, we get

$S_{1}^{d}-S_{1}^{\Lambda} \simeq-\frac{\sqrt{3}\left(\mu^{2}\right)^{d-3} a^{2}}{\pi}\left[\frac{1}{3-d}-\ln \frac{\Lambda^{2}}{\mu^{2}}+2.711355256\right]$.

Here, one encounters a subtlety: in Ref. [34], a different cutoff regularization is used at an intermediate step. Namely, instead of Eq. (123), one writes:

$$
\begin{aligned}
\tilde{S}_{1}^{\Lambda}= & -8 \pi a \int^{\Lambda} \frac{d^{3} \mathbf{k}}{(2 \pi)^{3}} \frac{1}{\mathbf{k}^{2}+\varepsilon^{2}}+\frac{1}{2}(8 \pi a)^{2} \int^{\Lambda} \frac{d^{3} \mathbf{k}}{(2 \pi)^{3}} \\
& \times \int^{\Lambda} \frac{d^{3} \mathbf{q}}{(2 \pi)^{3}} \frac{1}{\left(\mathbf{k}^{2}+\varepsilon^{2}\right)^{2}\left(\mathbf{k}^{2}+\mathbf{k} \mathbf{q}+\mathbf{q}^{2}+\varepsilon^{2}\right)}+\cdots .
\end{aligned}
$$

Carrying out explicit calculations, it can be shown that ${ }^{10}$

$$
\begin{aligned}
\tilde{S}_{1}^{\Lambda}-S_{1}^{\Lambda}= & -\frac{\sqrt{3} a^{2}}{\pi}\left[\frac{\sqrt{3}}{2 \pi} \ln 3+\frac{1}{2}-\frac{2}{\sqrt{3} \pi}\right. \\
& \left.+\frac{2}{\sqrt{3} \pi} \int_{0}^{1} d k \ln k\left(\frac{k+2}{k^{2}+k+1}-\frac{k-2}{k^{2}-k+1}\right)\right],
\end{aligned}
$$

and, therefore,

$$
\begin{aligned}
S_{1}^{d}-\tilde{S}_{1}^{\Lambda} \simeq & -\frac{\sqrt{3}\left(\mu^{2}\right)^{d-3} a^{2}}{\pi}\left[\frac{1}{3-d}-2 \ln N+2 \ln (\mu L)\right. \\
& -0.020508246] .
\end{aligned}
$$

Comparing with Ref. [34], one has to use the same prescription as there. Hence, Eq. (127) contains two correction factors: the one accounting for the different UV

\footnotetext{
${ }^{10}$ This result is interesting by its own. The above two expressions differ only in the ultraviolet region. In the quantity $S_{1}^{\Lambda}$, the limit $\Lambda \rightarrow \infty$ has been already performed in the internal bubble (as it is implied by the form of integral equations we are using), whereas in the quantity $\tilde{S}_{1}^{\Lambda}$ both cutoffs are held finite and equal. As expected, the choice of different prescriptions can be compensated by a finite renormalization of the coupling constant $H_{0}(\Lambda)$.
}

cutoffs used, and another that takes into account the difference between the cutoff and the dimensional regularizations.

Further, the analog of our quantity $\mathcal{R}$ in Ref. [34] is defined as

$$
\begin{aligned}
& \frac{1}{L^{6}} \sum_{\mathbf{k} \neq \mathbf{0}}^{\Lambda} \sum_{\mathbf{q}}^{\Lambda} \frac{1}{\mathbf{k}^{4}\left(\mathbf{k}^{2}+\mathbf{q}^{2}+(\mathbf{k}+\mathbf{q})^{2}\right)}-\frac{1}{L^{3}} \sum_{\mathbf{k} \neq \mathbf{0}}^{\Lambda} \frac{1}{\mathbf{k}^{4}} \int^{\Lambda} \frac{d^{3} \mathbf{q}}{2 \mathbf{q}^{2}} \\
& \quad=-\frac{\sqrt{3}}{32 \pi^{3}} \ln N-\frac{1}{(2 \pi)^{6}} \mathcal{R}_{1},
\end{aligned}
$$

where $N$ is the same as in Eq. (C2). The above expression can be further rewritten as

$$
\begin{aligned}
- & \frac{\sqrt{3}}{32 \pi^{3}} \ln N-\frac{1}{(2 \pi)^{6}} \mathcal{R}_{1} \\
= & \frac{1}{L^{3}} \sum_{\mathbf{k} \neq \mathbf{0}}^{\Lambda} \frac{1}{\mathbf{k}^{4}}\left(\frac{1}{L^{3}} \sum_{\mathbf{q}}^{\Lambda}-\int^{\Lambda} \frac{d^{3} \mathbf{q}}{(2 \pi)^{3}}\right) \frac{1}{2} \frac{1}{\mathbf{k}^{2}+\mathbf{k} \mathbf{q}+\mathbf{q}^{2}} \\
& +\frac{1}{2 L^{3}} \sum_{\mathbf{k} \neq \mathbf{0}}^{\Lambda} \frac{1}{\mathbf{k}^{4}} \int^{\Lambda} \frac{d^{3} \mathbf{q}}{(2 \pi)^{3}}\left(\frac{1}{\mathbf{k}^{2}+\mathbf{k q}+\mathbf{q}^{2}}-\frac{1}{\mathbf{q}^{2}}\right) .
\end{aligned}
$$

When $\Lambda \rightarrow \infty$, the first term converges to $\frac{1}{2(2 \pi)^{6}} \mathcal{G}$, where $\mathcal{G}$ is given by Eq. (C3). The evaluation of the integral in the second term gives:

$$
\begin{aligned}
& \frac{1}{2} \int d^{3} \mathbf{q}\left(\frac{1}{\mathbf{k}^{2}+\mathbf{k q}+\mathbf{q}^{2}}-\frac{1}{\mathbf{q}^{2}}\right) \\
& =-\sqrt{3} \pi^{2}|\mathbf{k}|+\left\{\left(\frac{\pi \Lambda^{2}}{|\mathbf{k}|}+\frac{\pi|\mathbf{k}|}{2}\right) \ln \frac{\Lambda^{2}+\Lambda|\mathbf{k}|+\mathbf{k}^{2}}{\Lambda^{2}-\Lambda|\mathbf{k}|+\mathbf{k}^{2}}-2 \pi \Lambda\right. \\
& \left.\quad+\sqrt{3} \pi|\mathbf{k}|\left(\arctan \frac{\sqrt{3}|\mathbf{k}|}{2 \Lambda+|\mathbf{k}|}+\arctan \frac{\sqrt{3}|\mathbf{k}|}{2 \Lambda-|\mathbf{k}|}\right)\right\} .
\end{aligned}
$$

One has to insert this expression into Eq. (129) and evaluate the sum over $\mathbf{k}$. Substituting the first term yields $-\frac{\sqrt{3}}{128 \pi^{4}}(\mathcal{L}+2 \pi \ln N)$, where $\mathcal{L}$ is given by Eq. (C2). Substituting the rest and performing the limit $\Lambda \rightarrow \infty$, one sees that the sum over $\mathbf{k}$ can be replaced by integration in this case. So, finally we get:

$$
\begin{aligned}
-\frac{\sqrt{3}}{32 \pi^{3}} \ln N-\frac{1}{(2 \pi)^{6}} \mathcal{R}_{1}= & \frac{1}{2(2 \pi)^{6}}\left(\mathcal{G}-\sqrt{3} \pi^{2} \mathcal{L}\right) \\
& -\frac{\sqrt{3}}{32 \pi^{3}} \ln N-\frac{1}{(2 \pi)^{6}} \chi
\end{aligned}
$$

where 


$$
\begin{aligned}
\chi= & \pi^{2}\left\{\frac{3}{2} \ln (3)-2+\frac{\sqrt{3} \pi}{2}\right. \\
& \left.+2 \int d x \ln (x)\left(\frac{x+2}{1+x+x^{2}}-\frac{x-2}{1-x+x^{2}}\right)\right\} \\
\simeq & -50.691124 .
\end{aligned}
$$

From this we finally get $\mathcal{R}_{1}=\frac{1}{2}\left(\mathcal{G}-\sqrt{3} \pi^{2} \mathcal{L}\right)-\chi$ and

$$
\mathcal{R}(\text { Ref. }[34]) \simeq \mathcal{R}_{1}+\sqrt{3} \pi^{3} \times 0.020508246 \simeq 19.186903,
$$

which agrees with the value given in Ref. [34].

Next, we calculate the term with two iterations of the driving term $Z$, which is an infinite-volume counterpart of the quantity $S_{2}$, considered in Sec. III C. In dimensional regularization, we have

$$
\begin{aligned}
S_{2}^{d}= & (8 \pi a)^{2} \int \frac{d^{d} \mathbf{k}}{(2 \pi)^{d}} \\
& \times \int \frac{d^{d} \mathbf{q}}{(2 \pi)^{d}} \frac{1}{\left(\mathbf{k}^{2}+\varepsilon^{2}\right)\left(\mathbf{k}^{2}+\mathbf{k q}+\mathbf{q}^{2}+\varepsilon^{2}\right)\left(\mathbf{k}^{2}+\varepsilon^{2}\right)} \\
& +\cdots,
\end{aligned}
$$

where the ellipses, as before, stand for the nonsingular terms at $\varepsilon \rightarrow 0$. Evaluating this expression, we obtain

$$
S_{2}^{d}=\frac{a^{2}\left(\mu^{2}\right)^{d-3}}{2 \pi}\left\{\frac{8 \pi}{3}\left(\frac{1}{3-d}+\Gamma^{\prime}(1)+\ln 4 \pi-\ln \frac{\varepsilon^{2}}{\mu^{2}}\right)+\mathcal{J}_{d}\right\},
$$

where

$$
\begin{aligned}
\mathcal{J}_{d} & =\int_{0}^{1} x d x\left\{\frac{2 \ln (g)+4}{\left(\frac{3}{2}-x\right) \sqrt{g}}-\frac{8}{x\left(\frac{3}{2}-x\right)} \arctan \frac{x}{2 \sqrt{g}}\right\} \\
& \simeq-9.436982652, \quad g=\frac{3-2 x-x^{2}}{4} .
\end{aligned}
$$

The counterpart of this integral in the cutoff regularization has already been calculated in Sec. III C. Using this expression, we have

$$
\begin{aligned}
& S_{2}^{d}-S_{2}^{\Lambda} \\
& =\frac{4 a^{2}\left(\mu^{2}\right)^{d-3}}{3}\left\{\frac{1}{3-d}+\Gamma^{\prime}(1)-\ln \frac{\Lambda^{2}}{\mu^{2}}+\frac{3}{8 \pi} \mathcal{J}_{d}-\frac{3}{4 \pi^{4}} \mathcal{I}_{0}\right\} \\
& \simeq \frac{4 a^{2}\left(\mu^{2}\right)^{d-3}}{3}\left\{\frac{1}{3-d}-2 \ln N+2 \ln (\mu L)+0.044721273\right\} .
\end{aligned}
$$

This enables one to relate the constants $\mathcal{Q}$ that appear in this work and in Ref. [34]:
$\mathcal{Q}($ Ref.[34] $) \simeq \mathcal{Q}+\frac{2 \pi^{4}}{3} 0.044721273 \simeq-102.1556055$

This value is quite close to the one quoted in Ref. [34].

\section{CONCLUSIONS AND OUTLOOK}

(i) Using the quantization condition derived in Refs. $[17,18,20]$, we derive the expressions for the energy shift of the three-particle ground-state energy level and the first excited level at order $L^{-6}$, and the particle-dimer ground state at order $L^{-3}$. This derivation provides a beautiful test of the formalism.

(ii) We carefully compare the result for the ground state to the expressions given in Refs. [8,9,16] and Ref. [34]. It has been shown that, apart from the relativistic corrections, which are absent in the present paper and in Ref. [34], all these expressions agree completely. The expressions for the excited levels and the particle-dimer level are new (the latter reproduces the well-known two-particle Lüscher formula at order $L^{-3}$ ).

(iii) The calculations for the higher excited levels can be done similarly to the first excited level. Also, the projection onto the different irreps of the octahedral groups can be treated in the same fashion.

(iv) A natural question arises here: how do our results change, if the higher-order two-body and three-body effective couplings, as well as mixing to the higher partial waves are taken into account? We argue that the final results, which are expressed through the threshold amplitude $\hat{\mathcal{M}}$, do not change at all. All the above effects are just included in this amplitude since, according to the power counting, none of them contributes up to and including order $L^{-6}$.

(v) From our results it is seen that the three-body force (encoded, in particular, in the coupling $\frac{H_{0}(\Lambda)}{\Lambda^{2}}$ ) starts to contribute at NNNLO to the three-particle energy levels, but appears already at leading order in the particle-dimer energy levels. Therefore, in order to measure, e.g., the three-pion force, it might be convenient to look at the $\pi \sigma$ scattering at higher quark masses, where $\sigma$ is a stable particle. With the use of a chiral extrapolation, one may then estimate the value of the three-pion coupling constant in the physical world.

\section{ACKNOWLEDGMENTS}

The authors would like to thank M. Hansen, F. RomeroLópez, S. Sharpe, C. Thomas and C. Urbach for interesting discussions. We would also like to thank S. Beane, W. Detmold and M. Savage for providing us with the notes of their calculations which made a detailed comparison of the 
results possible. H.W.H. and A. R. are grateful to the Mainz Institute for Theoretical Physics (MITP) for its hospitality and its partial support during the completion of this work. J. Y. P., J. W., U. G. M. and A. R. acknowledge the support from the DFG (CRC 110 "Symmetries and the Emergence of Structure in QCD"). U.G. M. and A. R. acknowledge support from Volkswagenstiftung under Contract No. 93562. The work of A. R. was in part supported by Shota Rustaveli National Science Foundation (SRNSF), Grant No. DI-2016-26. H. W. H. was supported by the Deutsche Forschungsgemeinschaft (DFG, German Research Foundation)-Projektnummer 279384907-SFB 1245 and the Federal Ministry of Education and Research (BMBF) under Contract No. 05P18RDFN1. The work of U. G. M. is supported in part by The Chinese Academy of Sciences (CAS) President's International Fellowship Initiative (PIFI) (Grant No. 2018DM0034).

\section{APPENDIX A: ENERGY SHIFT OF THREE-PARTICLE SCATTERING STATES}

The energy of the scattering states vanishes in the infinite volume limit. We quote their finite volume energy $E$ in terms of the quantity $\kappa^{2}=L^{2} m E /(2 \pi)^{2}$.

\section{Ground state $\left(A_{1}^{+}\right)$}

The energy shift of the ground state (which resides in the $A_{1}^{+}$irrep) is

$$
\kappa^{2}=\frac{g_{0}}{L}\left(1+\frac{g_{1}}{L}+\frac{g_{2}}{L^{2}}+\frac{g_{3}}{L^{3}} \ln \frac{m L}{2 \pi}+\frac{g_{4}}{L^{3}}+\cdots\right),
$$

with

$$
\begin{aligned}
& g_{0}=\frac{3}{\pi} a, \\
& g_{1}=2.837297480 a, \\
& g_{2}=9.725330808 a^{2}, \\
& g_{3}=8 \pi\left(\frac{2 \sqrt{3}}{\pi}-\frac{8}{3}\right) a^{3}, \\
& g_{4}=\left(-5.159159617+6 \pi\left(\frac{r}{a}\right)-8 \pi\left(\frac{\hat{\mathcal{M}}}{a^{2}}\right)\right) a^{3} .
\end{aligned}
$$

\section{The 1st excited state $\left(A_{1}^{+}\right)$}

The energy shift of the 1 st excited state in the $A_{1}^{+}$irrep is

$$
\kappa^{2}-1=\frac{h_{0}}{L}\left(1+\frac{h_{1}}{L}+\frac{h_{2}}{L^{2}}+\frac{h_{3}}{L^{3}} \ln \frac{m L}{2 \pi}+\frac{h_{4}}{L^{3}}+\cdots\right),
$$

with

$$
\begin{aligned}
& h_{0}=\frac{10}{\pi} a, \\
& h_{1}=0.279070 a, \\
& h_{2}=\left(8.494802+\frac{7 \pi^{2}}{5}\left(\frac{r}{a}\right)\right) a^{2}, \\
& h_{3}=\frac{27}{5} \times 8 \pi\left(\frac{2 \sqrt{3}}{\pi}-\frac{8}{3}\right) a^{3}, \\
& h_{4}=\left(-172.001650+83.745841\left(\frac{r}{a}\right)-\frac{27}{5} \times 8 \pi\left(\frac{\hat{\mathcal{M}}}{a^{2}}\right)\right) a^{3} .
\end{aligned}
$$

\section{The 1st excited state $\left(E^{+}\right)$}

The energy shift of the 1 st excited state in the $E^{+}$irrep is

$$
\kappa^{2}-1=\frac{h_{0}^{\prime}}{L}\left(1+\frac{h_{1}^{\prime}}{L}+\frac{h_{2}^{\prime}}{L^{2}}+\frac{h_{4}^{\prime}}{L^{3}}+\cdots\right),
$$

with

$$
\begin{aligned}
h_{0}^{\prime} & =\frac{4}{\pi} a, \\
h_{1}^{\prime} & =2.984094 a, \\
h_{2}^{\prime} & =\left(3.001706+\frac{\pi^{2}}{2}\left(\frac{r}{a}\right)\right) a^{2}, \\
h_{4}^{\prime} & =\left(-28.89478538+54.584571\left(\frac{r}{a}\right)\right) a^{3} .
\end{aligned}
$$

\section{APPENDIX B: TRANSFORMATIONS OF SUMS AND INTEGRALS}

\section{Derivation of Eq. (C2)}

The quantity $\mathcal{L}$ from Eq. (C2) can be rewritten as

$$
\begin{aligned}
\mathcal{L}= & \sum_{\mathbf{j} \neq \mathbf{0}}\left(\frac{1}{\mathbf{j}^{3}}-\frac{1}{\left(\mathbf{j}^{2}+1\right)^{3 / 2}}-\frac{3}{2} \frac{1}{\left(\mathbf{j}^{2}+1\right)^{5 / 2}}-\frac{15}{8} \frac{1}{\left(\mathbf{j}^{2}+1\right)^{7 / 2}}\right) \\
& +\lim _{N \rightarrow \infty}\left(\sum_{\mathbf{j}}^{N} \frac{1}{\left(\mathbf{j}^{2}+1\right)^{3 / 2}}-4 \pi \ln N-1\right) \\
& +\frac{3}{2}\left(\sum_{\mathbf{j}} \frac{1}{\left(\mathbf{j}^{2}+1\right)^{5 / 2}}-1\right) \\
& +\frac{15}{8}\left(\sum_{\mathbf{j}} \frac{1}{\left(\mathbf{j}^{2}+1\right)^{7 / 2}}-1\right),
\end{aligned}
$$

where we have already performed the limit $N \rightarrow \infty$ in the first and the third terms. Using the Poisson summation formula in the second and the third terms, we finally obtain 


$$
\begin{aligned}
\mathcal{L}= & \sum_{\mathbf{j} \neq \mathbf{0}}\left(\frac{1}{\mathbf{j}^{3}}-\frac{1}{\left(\mathbf{j}^{2}+1\right)^{3 / 2}}-\frac{3}{2} \frac{1}{\left(\mathbf{j}^{2}+1\right)^{5 / 2}}-\frac{15}{8} \frac{1}{\left(\mathbf{j}^{2}+1\right)^{7 / 2}}\right) \\
& +\pi(4 \ln 2-1)-\frac{35}{8}+4 \pi \sum_{\mathbf{j} \neq \mathbf{0}}\left(K_{0}(2 \pi|\mathbf{j}|)\right. \\
& \left.+\pi|\mathbf{j}| K_{1}(2 \pi|\mathbf{j}|)\right)+2 \pi^{2} \sum_{\mathbf{j} \neq \mathbf{0}}\left(K_{1}(2 \pi|\mathbf{j}|)\right. \\
& \left.+\pi|\mathbf{j}| K_{0}(2 \pi|\mathbf{j}|)\right),
\end{aligned}
$$

where $K_{0}(z), K_{1}(z)$ denote the modified Bessel functions of the second kind. Carrying out the summation in the above equation numerically, we finally arrive at the value given in Eq. (C2).

\section{Derivation of Eq. (C3)}

Using the Poisson summation formula, we get

$$
\mathcal{I}(\mathbf{n})=\pi \sum_{\mathbf{j} \neq \mathbf{0}} \frac{e^{i \pi \mathbf{n} \mathbf{j}}}{|\mathbf{j}|} \exp (-\sqrt{3} \pi|\mathbf{j}||\mathbf{n}|) .
$$

Carrying out the summation numerically, we arrive at the value given in Eq. (C3).

\section{Derivation of Eq. (33)}

Consider the quantity

$$
\begin{aligned}
\Sigma & =\frac{1}{L^{6}} \sum_{\mathbf{k} \neq \mathbf{0}}^{\Lambda} \sum_{\mathbf{q} \neq \mathbf{0}}^{\Lambda} \frac{1}{\mathbf{k}^{2}} \frac{1}{\mathbf{k}^{2}+\mathbf{q}^{2}+\mathbf{k q}} \frac{1}{\mathbf{q}^{2}} \\
& =\frac{1}{(2 \pi)^{6}} \sum_{\mathbf{j} \neq \mathbf{0}}^{N} \sum_{\mathbf{n} \neq \mathbf{0}}^{N} \frac{1}{\mathbf{j}^{2}} \frac{1}{\mathbf{j}^{2}+\mathbf{n}^{2}+\mathbf{j n} \mathbf{n}} \frac{1}{\mathbf{n}^{2}},
\end{aligned}
$$

where $\mathbf{j}, \mathbf{n} \in \mathbb{Z}^{3}$ and $N=\frac{\Lambda L}{2 \pi}$. We may further write $\Sigma=(\Sigma-\hat{\Sigma})+\hat{\Sigma}$, where

$$
\hat{\Sigma}=\frac{1}{(2 \pi)^{6}} \int_{1}^{N} \frac{d^{3} \mathbf{j}}{\mathbf{j}^{2}} \int_{1}^{N} \frac{d^{3} \mathbf{n}}{\mathbf{n}^{2}} \frac{1}{\mathbf{j}^{2}+\mathbf{n}^{2}+\mathbf{j n}}
$$

After angular integration, this integral takes the form

$$
\hat{\Sigma}=\frac{1}{4 \pi^{4}} \int_{1}^{N} \int_{1}^{N} \frac{d x d y}{x y} \ln \frac{x^{2}+y^{2}+x y}{x^{2}+y^{2}-x y} .
$$

Using partial integration, it is possible to perform the integral over the variable $y$ analytically. The result is given by:

$$
\begin{aligned}
\hat{\Sigma}= & \frac{\ln N}{2 \pi^{4}} \int_{1 / N}^{1} \frac{d x}{x} \ln \frac{x^{2}+x+1}{x^{2}-x+1} \\
& +\frac{1}{2 \pi^{4}} \int_{1 / N}^{1} \frac{d x}{x} \ln x \ln \frac{x^{2}+x+1}{x^{2}-x+1} .
\end{aligned}
$$

For large values of $N$, one may replace $1 / N$ by zero. The first integral then equals $\pi^{2} / 6$, and we get

$$
\begin{aligned}
\hat{\Sigma} & =\frac{1}{12 \pi^{2}} \ln N+\frac{1}{2 \pi^{4}} \int_{0}^{1} \frac{d x}{x} \ln x \ln \frac{x^{2}+x+1}{x^{2}-x+1} \\
& =\frac{1}{12 \pi^{2}} \ln N+\text { const. }
\end{aligned}
$$

The constant does not play any role and can be dropped. Performing the limit $N \rightarrow \infty$ in the quantity $\Sigma-\hat{\Sigma}$, we finally arrive at Eq. (33).

\section{Derivation of Eq. (C4)}

The infinite sum that enters Eq. (C4) can be rewritten in the following way:

$$
\begin{aligned}
\Sigma= & \sum_{\mathbf{j} \neq \mathbf{0}}^{N} \sum_{\mathbf{n} \neq \mathbf{0}}^{N} \frac{1}{\mathbf{j}^{2}} \frac{1}{\mathbf{j}^{2}+\mathbf{j n}+\mathbf{n}^{2}} \frac{1}{\mathbf{n}^{2}} \\
= & \sum_{\mathbf{j} \neq \mathbf{0}}^{N} \sum_{\mathbf{n} \neq \mathbf{0}}^{N} \frac{1}{\mathbf{j}^{2}\left(\mathbf{j}^{2}+1\right)^{3}} \frac{1}{\mathbf{j}^{2}+\mathbf{j n}+\mathbf{n}^{2}} \frac{1}{\mathbf{n}^{2}\left(\mathbf{n}^{2}+1\right)^{3}}+2 \sum_{\mathbf{j} \neq \mathbf{0}}^{N} \sum_{\mathbf{n} \neq \mathbf{0}}^{N} \varphi(\mathbf{j}) \frac{1}{\left(\mathbf{j}^{2}+\mathbf{j n}+\mathbf{n}^{2}\right)\left(\mathbf{j}^{2}+\mathbf{j n}+\mathbf{n}^{2}+1\right)^{3}} \frac{1}{\mathbf{n}^{2}\left(\mathbf{n}^{2}+1\right)^{3}} \\
& +2 \sum_{\mathbf{j} \neq \mathbf{0}}^{N} \sum_{\mathbf{n} \neq \mathbf{0}}^{N} \varphi(\mathbf{j}) h(\mathbf{j}, \mathbf{n}) \frac{1}{\mathbf{n}^{2}\left(\mathbf{n}^{2}+1\right)^{3}}+\sum_{\mathbf{j} \neq \mathbf{0}}^{N} \sum_{\mathbf{n} \neq \mathbf{0}}^{N} \varphi(\mathbf{j}) \frac{1}{\left(\mathbf{j}^{2}+\mathbf{j n}+\mathbf{n}^{2}\right)\left(\mathbf{j}^{2}+\mathbf{j n}+\mathbf{n}^{2}+1\right)^{3}} \varphi(\mathbf{n}) \\
& +\sum_{\mathbf{j} \neq \mathbf{0}}^{N} \sum_{\mathbf{n} \neq \mathbf{0}}^{N} \varphi(\mathbf{j}) h(\mathbf{j}, \mathbf{n}) \varphi(\mathbf{n}),
\end{aligned}
$$

where 


$$
\begin{aligned}
\varphi(\mathbf{n}) & =\frac{1}{\mathbf{n}^{2}+1}+\frac{1}{\left(\mathbf{n}^{2}+1\right)^{2}}+\frac{1}{\left(\mathbf{n}^{2}+1\right)^{3}}, \\
h(\mathbf{j}, \mathbf{n}) & =\frac{1}{\mathbf{j}^{2}+\mathbf{j n}+\mathbf{n}^{2}+1}+\frac{1}{\left(\mathbf{j}^{2}+\mathbf{j n}+\mathbf{n}^{2}+1\right)^{2}}+\frac{1}{\left(\mathbf{j}^{2}+\mathbf{j n}+\mathbf{n}^{2}+1\right)^{3}} .
\end{aligned}
$$

The first, second and fourth terms in Eq. (B9) can be grouped together. We denote the sum of these three terms by $\Sigma_{1}$. Further, in the third and the fifth terms of the same equation, we add and subtract the terms with $\mathbf{j}=\mathbf{0}$ or $\mathbf{n}=\mathbf{0}$. As a result, we obtain $\Sigma=\Sigma_{1}+\Sigma_{2}+\Sigma_{3}+\Sigma_{4}$, where the individual terms take the following form

$$
\begin{aligned}
& \Sigma_{1}=\sum_{\mathbf{j} \neq \mathbf{0}}^{N} \sum_{\mathbf{n} \neq \mathbf{0}}^{N}\left\{\frac{1}{\mathbf{j}^{2}\left(\mathbf{j}^{2}+1\right)^{3}} \frac{1}{\mathbf{j}^{2}+\mathbf{j n}+\mathbf{n}^{2}} \frac{1}{\mathbf{n}^{2}\left(\mathbf{n}^{2}+1\right)^{3}}+\varphi(\mathbf{j}) \frac{1}{\left(\mathbf{j}^{2}+\mathbf{j n}+\mathbf{n}^{2}\right)\left(\mathbf{j}^{2}+\mathbf{j n}+\mathbf{n}^{2}+1\right)^{3}} \frac{1}{\mathbf{n}^{2}}\left(1+\frac{1}{\left(\mathbf{n}^{2}+1\right)^{3}}\right)\right\}, \\
& \Sigma_{2}=-6 \sum_{\mathbf{n} \neq \mathbf{0}}^{N} \frac{1}{\mathbf{n}^{2}} \varphi(\mathbf{n})-27 \\
& \Sigma_{3}=2 \sum_{\mathbf{n} \neq \mathbf{0}}^{N} \sum_{\mathbf{j}}^{N} \varphi(\mathbf{j}) h(\mathbf{j}, \mathbf{n}) \frac{1}{\mathbf{n}^{2}\left(\mathbf{n}^{2}+1\right)^{3}}, \\
& \Sigma_{4}=\sum_{\mathbf{n}}^{N} \sum_{\mathbf{j}}^{N} \varphi(\mathbf{j}) h(\mathbf{j}, \mathbf{n}) \varphi(\mathbf{n}) .
\end{aligned}
$$

$\Sigma_{1}$ and $\Sigma_{2}$ are rapidly converging sums. We may perform the limit $N \rightarrow \infty$ in these terms, obtaining

$$
\Sigma_{1} \simeq 2.8391985, \quad \Sigma_{2} \simeq-120.8804992739 .
$$

In order to calculate $\Sigma_{3}$ and $\Sigma_{4}$, the Poisson summation method is used. The quantity $\Sigma_{3}$ is equal to

$$
\Sigma_{3}=2 \sum_{\mathbf{n}} \frac{1}{\mathbf{n}^{2}\left(\mathbf{n}^{2}+1\right)^{3}} \Psi(\mathbf{n}),
$$

where

$$
\Psi(\mathbf{n})=\sum_{\mathbf{j}} \int d^{3} \mathbf{k} e^{2 \pi i \mathbf{k} \mathbf{j}} \varphi(\mathbf{k}) h(\mathbf{k}, \mathbf{n})=\sum_{a=1}^{3} \sum_{b=1}^{3} I_{a b},
$$

and

$$
\begin{aligned}
I_{a b} & =\int d^{3} \mathbf{k} e^{2 \pi i \mathbf{k} \mathbf{j}} \frac{1}{\left(\mathbf{k}^{2}+1\right)^{a}\left(\mathbf{k}^{2}+\mathbf{k n}+\mathbf{n}^{2}+1\right)^{b}} \\
& =\frac{\Gamma(a+b)}{\Gamma(a) \Gamma(b)} \int_{0}^{1} d x e^{-i \pi x \mathbf{n} \mathbf{j}} \int d^{3} \mathbf{k} e^{2 \pi i \mathbf{k} \mathbf{j}} \frac{x^{b-1}(1-x)^{a-1}}{\left(f^{2}+\mathbf{k}^{2}\right)^{a+b}} \\
& =\frac{1}{\Gamma(a) \Gamma(b)} \int_{0}^{1} d x e^{-i \pi x \mathbf{n} \mathbf{j}} x^{b-1}(1-x)^{a-1} \int_{0}^{\infty} d \lambda \lambda^{a+b-1} \int d^{3} \mathbf{k} e^{2 \pi i \mathbf{k} \mathbf{j}-\lambda\left(f^{2}+\mathbf{k}^{2}\right)} \\
& =\frac{\pi^{3 / 2}}{\Gamma(a) \Gamma(b)} \int_{0}^{1} d x \cos (\pi x \mathbf{n} \mathbf{j}) x^{b-1}(1-x)^{a-1} \int_{0}^{\infty} d \lambda \lambda^{a+b-5 / 2} \int e^{-\lambda f^{2}-\pi^{2} \mathbf{j}^{2} / \lambda} .
\end{aligned}
$$

Here,

$$
f^{2}=1+\left(x-\frac{x^{2}}{4}\right) \mathbf{n}^{2} .
$$


Note that we have set $N \rightarrow \infty$ everywhere, since all integrals and sums are convergent. Using the above equations, we finally get:

$$
\Sigma_{3}=2 \pi^{3 / 2} \sum_{\mathbf{n} \neq \mathbf{0}} \sum_{\mathbf{j}} \frac{1}{\mathbf{n}^{2}\left(\mathbf{n}^{2}+1\right)^{3}} \int_{0}^{1} d x \cos (\pi x \mathbf{n} \mathbf{j}) \int_{0}^{\infty} \frac{d \lambda}{\sqrt{\lambda}} e^{-\lambda f^{2}-\pi^{2} \mathbf{j}^{2} / \lambda} \times\left(1+\lambda x+\frac{\lambda^{2} x^{2}}{2}\right)\left(1+\lambda(1-x)+\frac{\lambda^{2}(1-x)^{2}}{2}\right) .
$$

Evaluating this expression numerically, we get

$$
\Sigma_{3} \simeq 27.2953+0.9312 \simeq 28.2266,
$$

where the first and the second terms correspond to $\mathbf{j}=\mathbf{0}$ and $\mathbf{j} \neq \mathbf{0}$ in the sum over all $\mathbf{j}$.

We use the same technique to calculate $\Sigma_{4}$. The result is given by:

$$
\Sigma_{4}=\sum_{\mathbf{n}} \sum_{\mathbf{j}} \sum_{a=1}^{3} \sum_{b=1}^{3} \sum_{c=1}^{3} I_{a b c}
$$

where

$$
\begin{aligned}
I_{a b c}= & \int^{N} \int^{N} d^{3} \mathbf{q} d^{3} \mathbf{k} e^{2 \pi i(\mathbf{j k}+\mathbf{q n})} \frac{1}{\left(\mathbf{q}^{2}+1\right)^{a}\left(\mathbf{k}^{2}+1\right)^{b}\left(\mathbf{q}^{2}+\mathbf{q} \mathbf{k}+\mathbf{k}^{2}+1\right)^{c}} \\
= & \frac{\Gamma(a+b+c)}{\Gamma(a) \Gamma(b) \Gamma(c)} \int_{0}^{\infty} d x d y d z \delta(1-x-y-z) x^{a-1} y^{b-1}(1-x-y)^{c-1} \\
& \times \int^{N} \int^{N} d^{3} \mathbf{q} d^{3} \mathbf{k} \frac{e^{2 \pi i(\mathbf{j k}+\mathbf{q n})}}{\left(1+(1-y) \mathbf{q}^{2}+(1-x) \mathbf{k}^{2}+(1-x-y) \mathbf{q} \mathbf{k}\right)^{a+b+c}} .
\end{aligned}
$$

The above expression is finite in the limit $N \rightarrow \infty$, except the term with $a=b=c=1$ and $\mathbf{n}=\mathbf{j}=0$. We can single out this divergent term and calculate the rest, where the limits of integration can be set to infinity. Performing the change of the variables

$$
\mathbf{q}=\frac{1}{\sqrt{1-y}}\left(\frac{\mathbf{u}}{\sqrt{2(1+\alpha)}}+\frac{\mathbf{v}}{\sqrt{2(1-\alpha)}}\right), \quad \mathbf{k}=\frac{1}{\sqrt{1-x}}\left(\frac{\mathbf{u}}{\sqrt{2(1+\alpha)}}-\frac{\mathbf{v}}{\sqrt{2(1-\alpha)}}\right)
$$

where

$$
\alpha=\frac{1-x-y}{2 \sqrt{1-x} \sqrt{1-y}}
$$

we get

$$
I_{a b c}=\frac{\Gamma(a+b+c)}{\Gamma(a) \Gamma(b) \Gamma(c)} \int_{0}^{\infty} d x d y d z \delta(1-x-y-z) \frac{x^{a-1} y^{b-1}(1-x-y)^{c-1}}{\left((1-x)(1-y)\left(1-\alpha^{2}\right)\right)^{3 / 2}} \times \int d^{6} \mathbf{Q} \frac{e^{2 \pi i \mathbf{Q R}}}{\left(1+\mathbf{Q}^{2}\right)^{a+b+c}},
$$

where we have defined the vectors in the 6-dimensional space

$$
\begin{aligned}
& \mathbf{Q}=(\mathbf{u}, \mathbf{v}) \\
& \mathbf{R}=\left(\frac{1}{\sqrt{2(1+\alpha)}}\left(\frac{\mathbf{j}}{\sqrt{1-x}}+\frac{\mathbf{n}}{\sqrt{1-y}}\right), \frac{1}{\sqrt{2(1-\alpha)}}\left(\frac{\mathbf{j}}{\sqrt{1-x}}-\frac{\mathbf{n}}{\sqrt{1-y}}\right)\right) .
\end{aligned}
$$

Carrying out the integration over $d^{6} \mathbf{Q}$ by exponentiating the denominator, we get 
$I_{a b c}=\frac{\pi^{3}}{\Gamma(a) \Gamma(b) \Gamma(c)} \int_{0}^{\infty} d x d y d z \delta(1-x-y-z) \frac{x^{a-1} y^{b-1}(1-x-y)^{c-1}}{\left((1-x)(1-y)\left(1-\alpha^{2}\right)\right)^{3 / 2}} \times \int d \lambda \lambda^{a+b+c-4} \exp \left(-\lambda-\pi^{2} \mathbf{R}^{2} / \lambda\right)$,

where

$$
\mathbf{R}^{2}=\left(\frac{\mathbf{j}^{2}}{1-x}+\frac{\mathbf{n}^{2}}{1-y}\right) \frac{1}{1-\alpha^{2}}-\frac{2 \alpha \mathbf{j n}}{\sqrt{1-x} \sqrt{1-y}\left(1-\alpha^{2}\right)}
$$

The infinite-volume integral is considered in Eq. (34). Adding everything together, we get

$$
\begin{aligned}
\lim _{N \rightarrow \infty}\left(\Sigma_{4}-\frac{8 \pi^{4}}{3} \ln N\right)= & \sum_{\mathbf{j}} \sum_{\mathbf{n}}\left(1-\delta_{\mathbf{j} \mathbf{0}} \delta_{\mathbf{n} \mathbf{0}}\right) \int_{0}^{\infty} d x d y d z \delta(1-x-y-z) \\
& \times \int_{0}^{\infty} \frac{d \lambda}{\lambda} \frac{\pi^{3} \exp \left(-\lambda-\pi^{2} \mathbf{R}^{2} / \lambda\right)}{\left((1-x)(1-y)\left(1-\alpha^{2}\right)\right)^{3 / 2}}\left(1+\lambda x+\frac{\lambda^{2} x^{2}}{2}\right) \\
& \times\left(1+\lambda y+\frac{\lambda^{2} y^{2}}{2}\right)\left(1+\lambda(1-x-y)+\frac{\lambda^{2}(1-x-y)^{2}}{2}\right) \\
& +\int_{0}^{\infty} d x d y d z \delta(1-x-y-z) \int_{0}^{\infty} \frac{d \lambda}{\lambda} \frac{\lambda^{3} \exp (-\lambda)}{\left((1-x)(1-y)\left(1-\alpha^{2}\right)\right)^{3 / 2}} \\
& \times\left\{\left(1+\lambda x+\frac{\lambda^{2} x^{2}}{2}\right)\left(1+\lambda y+\frac{\lambda^{2} y^{2}}{2}\right)\right. \\
& \left.\times\left(1+\lambda(1-x-y)+\frac{\lambda^{2}(1-x-y)^{2}}{2}\right)-1\right\}+\mathcal{I}_{0} \\
\simeq & 150.15376281+25.1433615+4.705512 \simeq-120.304889 .
\end{aligned}
$$

Here, the first, second and the third terms correspond to: $\mathbf{j}=\mathbf{n}=\mathbf{0}$; either $\mathbf{j}=\mathbf{0}$ or $\mathbf{n}=\mathbf{0}$; both $\mathbf{j}, \mathbf{n} \neq \mathbf{0}$.

Adding everything, we arrive at the numerical value given in Eq. (C4).

\section{Derivation of Eq. (34)}

Carrying out the integration over the angles and rescaling the integration variables, the 1.h.s. of Eq. (34) takes the form

$$
I_{\varepsilon}=\frac{a^{2}}{8 \pi^{4}} \int_{0}^{x} \frac{p d p}{p^{2}+1} \int_{0}^{x} \frac{q d q}{q^{2}+1} \ln \frac{p^{2}+p q+q^{2}+1}{p^{2}-p q+q^{2}+1}
$$

where $x=\Lambda / \varepsilon$. Performing the partial integration and rescaling the integration variables in some terms, we arrive at

$$
\begin{aligned}
I_{\varepsilon}= & \frac{a^{2}}{8 \pi^{4}} \ln x \int_{x^{-1}}^{1} \frac{q d q}{q^{2}+x^{-2}} \ln \frac{q^{2}+q+1+x^{-2}}{q^{2}-q+1+x^{-2}} \\
& -\frac{a^{2}}{16 \pi^{4}} \int_{0}^{x} d p \ln \left(p^{2}+1\right) \int_{0}^{x} \frac{q d q}{q^{2}+1}\left[\frac{2 p+q}{p^{2}+p q+q^{2}+1}-\frac{2 p-q}{p^{2}-p q+q^{2}+1}\right] .
\end{aligned}
$$

The integral over the variable $q$ in the second term can be performed analytically. Neglecting everywhere the terms that vanish at $x \rightarrow \infty$, we finally get:

$$
I_{\varepsilon}=\frac{a^{2}}{24 \pi^{2}} \ln x+\frac{a^{2}}{8 \pi^{4}} \int_{0}^{1} d p \frac{\ln p}{p} \ln \frac{p^{2}+p+1}{p^{2}-p+1}-\frac{a^{2}}{16 \pi^{3}} \int_{0}^{\infty} d p \frac{\ln \left(p^{2}+1\right)}{p^{2}+1}\left[1-\frac{1}{\sqrt{4+3 p^{2}}}\right] .
$$


From this expression, one can read off the expression for the numerical constant $\mathcal{I}_{0}$ in Eq. (34):

$$
\begin{aligned}
\mathcal{I}_{0}= & 8 \pi^{2} \int_{0}^{1} d p \frac{\ln p}{p} \ln \frac{p^{2}+p+1}{p^{2}-p+1} \\
& -4 \pi^{3} \int_{0}^{\infty} d p \frac{\ln \left(p^{2}+1\right)}{p^{2}+1}\left[1-\frac{1}{\sqrt{4+3 p^{2}}}\right] \\
\simeq & -375.754658 .
\end{aligned}
$$

\section{APPENDIX C: INFINITE SUMS}

In this Appendix, we collect numerical values for all infinite sums over integer numbers, which appear in the main text. In the following, $\mathbf{j}_{0}=(0,0,1)$ is a fixed unit vector, and the sum over the unit vector $\mathbf{i}_{0}$ runs over six possible orientations on the cubic lattice. The method of calculation of some of these sums is considered in Appendix B, and the remaining sums are calculated along the same pattern.

To calculate the energy shift of the ground state, we need the sums over the single integer vector:

$$
\begin{aligned}
& \mathcal{I}=\lim _{N \rightarrow \infty}\left\{\sum_{\mathbf{j} \neq \mathbf{0}}^{N} \frac{1}{\mathbf{j}^{2}}-4 \pi N\right\} \simeq-8.91363291781 \\
& \mathcal{J}=\sum_{\mathbf{j} \neq \mathbf{0}}^{\infty} \frac{1}{\mathbf{j}^{4}} \simeq 16.532315959 \\
& \mathcal{K}=\sum_{\mathbf{j} \neq \mathbf{0}}^{\infty} \frac{1}{\mathbf{j}^{6}} \simeq 8.401923974433
\end{aligned}
$$

and

$$
\mathcal{L}=\lim _{N \rightarrow \infty}\left[\sum_{\mathbf{j} \neq \mathbf{0}}^{N} \frac{1}{|\mathbf{j}|^{3}}-4 \pi \ln N\right] \simeq 3.82192350,
$$

and

$$
\mathcal{G}=\sum_{\mathbf{n} \neq \mathbf{0}} \frac{1}{\mathbf{n}^{4}} \tilde{\mathcal{I}}(\mathbf{n}) \simeq 0.123259025
$$

as well as the sums over two integer vectors:

$$
\begin{aligned}
\mathcal{Q} & =\lim _{N \rightarrow \infty}\left[\frac{1}{2} \sum_{\mathbf{j} \neq \mathbf{0}}^{N} \sum_{\mathbf{n} \neq \mathbf{0}}^{N} \frac{1}{\mathbf{j}^{2}} \frac{1}{\mathbf{j}^{2}+\mathbf{j n}+\mathbf{n}^{2}} \frac{1}{\mathbf{n}^{2}}-\frac{4 \pi^{4}}{3} \ln N\right] \\
& \simeq-105.0597779 .
\end{aligned}
$$

In the expansion of the energy shift of the first excited state in the $A_{1}^{+}$irrep, the following sums over a single integer vector appear (here, $\mathbf{j}_{0}$ denotes an integer vector with $\left.\left|\mathbf{j}_{0}\right|=1\right)$ :

$$
\begin{aligned}
& \mathcal{I}_{1}=\lim _{N \rightarrow \infty}\left(\sum_{|\mathbf{j}| \neq 1}^{N} \frac{1}{\mathbf{j}^{2}-1}-4 \pi N\right) \simeq-1.2113357 \\
& \mathcal{J}_{1}=\sum_{|\mathbf{j}| \neq 1} \frac{1}{\left(\mathbf{j}^{2}-1\right)^{2}} \simeq 23.2432219 \\
& \mathcal{K}_{1}=\sum_{|\mathbf{j}| \neq 1} \frac{1}{\left(\mathbf{j}^{2}-1\right)^{3}} \simeq 13.0593768 \\
& \mathcal{I}_{2}=\lim _{N \rightarrow \infty}\left(\sum_{\mathbf{j} \neq \mathbf{0},-\mathbf{j}_{0}}^{N} \frac{1}{\mathbf{j}^{2}+\mathbf{j} \mathbf{j}_{0}}-4 \pi N\right) \simeq-6.37480912 \\
& \mathcal{J}_{2}=\sum_{\mathbf{j} \neq \mathbf{0},-\mathbf{j}_{0}} \frac{1}{\left(\mathbf{j}^{2}+\mathbf{j} \mathbf{j}_{0}\right)^{2}} \simeq 18.343774 \\
& \mathcal{K}_{2}=\sum_{\mathbf{j} \neq \mathbf{0},-\mathbf{j}_{0}} \frac{1}{\left(\mathbf{j}^{2}+\mathbf{j} \mathbf{j}_{0}\right)^{3}} \simeq 10.2376434
\end{aligned}
$$

and

$$
\begin{aligned}
& \mathcal{J}_{11}=\sum_{|\mathbf{j}| \neq 0,1}^{\infty} \frac{1}{\left(\mathbf{j}^{2}-1\right)^{2}} \simeq 22.2432219 \\
& \mathcal{J}_{12}=\sqrt{6} \sum_{|\mathbf{j}| \neq 0,1}^{\infty} \frac{1}{\mathbf{j}^{2}-1} \frac{1}{\mathbf{j}^{2}+\mathbf{j} \mathbf{j}_{0}} \simeq 40.3648902,
\end{aligned}
$$

$$
\mathcal{J}_{22}=\sum_{\left|\mathbf{i}_{0}\right|=1} \sum_{|\mathbf{j}| \neq 0,1}^{\infty} \frac{1}{\mathbf{i}_{0} \mathbf{j}+\mathbf{j}^{2}} \frac{1}{\mathbf{j}^{2}+\mathbf{j} \mathbf{j}_{0}} \simeq 76.3433459 \text {, }
$$




$$
\begin{gathered}
\mathcal{L}_{11}=\lim _{N \rightarrow \infty}\left(\sum_{|\mathbf{j}| \neq 0,1}^{N} \frac{\sqrt{\mathbf{j}^{2}-\frac{4}{3}}}{\left.\mathbf{j}^{2}-1\right)^{2}}-4 \pi \ln N\right) \simeq 7.153930983 \\
\mathcal{L}_{12}=\sqrt{6} \lim _{N \rightarrow \infty}\left(\sum_{|\mathbf{j}| \neq 0,1}^{N} \frac{\sqrt{\mathbf{j}^{2}-\frac{4}{3}}}{\left(\mathbf{j}^{2}-1\right)\left(\mathbf{j}^{2}+\mathbf{j} \mathbf{j}_{0}\right)}-4 \pi \ln N\right) \simeq 3.048537581, \\
\mathcal{L}_{22}=\sum_{\left|\mathbf{i}_{0}\right|=1} \lim _{N \rightarrow \infty}\left(\sum_{|\mathbf{j}| \neq 0,1}^{N} \frac{\sqrt{\mathbf{j}^{2}-\frac{4}{3}}}{\left(\mathbf{i}_{0} \mathbf{j}+\mathbf{j}^{2}\right)\left(\mathbf{j}^{2}+\mathbf{j} \mathbf{j}_{0}\right)}-4 \pi \ln N\right) \simeq-17.27264446, \\
\mathcal{G}_{11}=\sum_{|\mathbf{j}| \neq 0,1}^{\infty} \frac{\mathcal{I}(\mathbf{j})}{\left(\mathbf{j}^{2}-1\right)^{2}} \simeq-1.028613861 \\
\mathcal{G}_{12}=\sqrt{6} \sum_{|\mathbf{j}| \neq 0,1}^{\infty} \frac{\mathcal{I}(\mathbf{j})}{\left(\mathbf{j}^{2}-1\right)\left(\mathbf{j}^{2}+\mathbf{j} \mathbf{j}_{0}\right)} \simeq-1.5491996719, \\
\mathcal{G}_{22}=\sum_{\left|\mathbf{i}_{0}\right|=1} \sum_{|\mathbf{j}| \neq 0,1}^{\infty} \frac{\mathcal{I}(\mathbf{j})}{\left(\mathbf{i}_{0} \mathbf{j}+\mathbf{j}^{2}\right)\left(\mathbf{j}^{2}+\mathbf{j} \mathbf{j}_{0}\right)} \simeq-2.3361490578,
\end{gathered}
$$

as well as the sums over two integer vectors:

$$
\begin{aligned}
& \mathcal{Q}_{11}=\lim _{N \rightarrow \infty}\left(\frac{1}{2} \sum_{|\mathbf{j}| \neq 0,1}^{N} \sum_{|\mathbf{n}| \neq 0,1}^{N} \frac{1}{\mathbf{j}^{2}-1} \frac{1}{\mathbf{j}^{2}+\mathbf{j n}+\mathbf{n}^{2}-1} \frac{1}{\mathbf{n}^{2}-1}-\frac{4 \pi^{4}}{3} \ln N\right) \simeq-39.5306, \\
& \mathcal{Q}_{12}=\sqrt{6} \lim _{N \rightarrow \infty}\left(\frac{1}{2} \sum_{|\mathbf{j}| \neq 0,1}^{N} \sum_{|\mathbf{n}| \neq 0,1}^{N} \frac{1}{\mathbf{j}^{2}-1} \frac{1}{\mathbf{j}^{2}+\mathbf{j n}+\mathbf{n}^{2}-1} \frac{1}{\mathbf{n}^{2}+\mathbf{n} \mathbf{j}_{0}}-\frac{4 \pi^{4}}{3} \ln N\right) \simeq-219.3579, \\
& \mathcal{Q}_{22}=\sum_{\left|\mathbf{i}_{0}\right|=1} \lim _{N \rightarrow \infty}\left(\frac{1}{2} \sum_{|\mathbf{j}| \neq 0,1}^{N} \sum_{|\mathbf{n}| \neq 0,1}^{N} \frac{1}{\mathbf{i}_{0} \mathbf{j}+\mathbf{j}^{2}} \frac{1}{\mathbf{j}^{2}+\mathbf{j n}+\mathbf{n}^{2}-1} \frac{1}{\mathbf{n}^{2}+\mathbf{n} \mathbf{j}_{0}}-\frac{4 \pi^{4}}{3} \ln N\right) \simeq-783.596 .
\end{aligned}
$$

Finally, the sums over the single and two integer vectors, appearing in the expression of the energy shift of the first excited level in the irrep $E^{+}$, are equal to:

$$
\begin{gathered}
\mathcal{J}_{22}^{\prime}=\sum_{\left|\mathbf{i}_{0}\right|=1} \lambda\left(\mathbf{i}_{0}\right) \sum_{|\mathbf{j}| \neq 0,1}^{\infty} \frac{1}{\mathbf{i}_{0} \mathbf{j}+\mathbf{j}^{2}} \frac{1}{\mathbf{j}^{2}+\mathbf{j} \mathbf{j}_{0}} \simeq 0.278429651 \\
\mathcal{K}_{22}^{\prime}=\frac{1}{2} \sum_{\left|\mathbf{i}_{0}\right|=1} \lambda\left(\mathbf{i}_{0}\right) \sum_{|\mathbf{j}| \neq 0,1}^{\infty}\left(\frac{1}{\left(\mathbf{i}_{0} \mathbf{j}+\mathbf{j}^{2}\right)^{2}} \frac{1}{\mathbf{j}^{2}+\mathbf{j} \mathbf{j}_{0}}+\frac{1}{\mathbf{i}_{0} \mathbf{j}+\mathbf{j}^{2}} \frac{1}{\left(\mathbf{j}^{2}+\mathbf{j} \mathbf{j}_{0}\right)^{2}}\right) \simeq 0.449738111, \\
\mathcal{L}_{22}^{\prime}=\sum_{\left|\mathbf{i}_{0}\right|=1} \lambda\left(\mathbf{i}_{0}\right) \sum_{|\mathbf{j}| \neq 0,1}^{\infty} \frac{\sqrt{\mathbf{j}^{2}-\frac{4}{3}}}{\left(\mathbf{i}_{0} \mathbf{j}+\mathbf{j}^{2}\right)\left(\mathbf{j}^{2}+\mathbf{j} \mathbf{j}_{0}\right)} \simeq 0.2888875370, \\
\mathcal{G}_{22}^{\prime}=\sum_{\left|\mathbf{i}_{0}\right|=1} \lambda\left(\mathbf{i}_{0}\right) \sum_{|\mathbf{j}| \neq 0,1}^{\infty} \frac{\mathcal{I}(\mathbf{j})}{\left(\mathbf{i}_{0} \mathbf{j}+\mathbf{j}^{2}\right)\left(\mathbf{j}^{2}+\mathbf{j} \mathbf{j}_{0}\right)} \simeq-0.018431808, \\
\frac{1}{2} \sum_{\left|\mathbf{i}_{0}\right|=1} \lambda\left(\mathbf{i}_{0}\right) \sum_{|\mathbf{j}| \neq 0,1}^{\infty} \sum_{|\mathbf{n}| \neq 0,1}^{\infty} \frac{1}{\mathbf{i}_{0} \mathbf{j}+\mathbf{j}^{2}} \frac{1}{\mathbf{j}^{2}+\mathbf{j} \mathbf{n}+\mathbf{n}^{2}-1} \frac{1}{\mathbf{n}^{2}+\mathbf{n} \mathbf{j}_{0}} \simeq 0.07825 .
\end{gathered}
$$


Here,

$$
\lambda\left(\mathbf{i}_{0}\right)=\left\{\begin{array}{ll}
1, & \text { if } \mathbf{i}_{0}=(0,0, \pm 1) \\
-\frac{1}{2}, & \text { otherwise }
\end{array},\right.
$$

and the integer vector $\mathbf{j}$ again belongs to the shell $r$. Note the absence of the logarithmic divergences in Eqs. (C11)-(C15).

[1] K. Polejaeva and A. Rusetsky, Eur. Phys. J. A 48, 67 (2012).

[2] U.-G. Meißner, G.-Rios, and A. Rusetsky, Phys. Rev. Lett. 114, 091602 (2015); 117, 069902(E) (2016).

[3] P. Guo, Phys. Rev. D 95, 054508 (2017).

[4] P. Guo and V. Gasparian, Phys. Lett. B 774, 441 (2017).

[5] R. A. Briceño and Z. Davoudi, Phys. Rev. D 87, 094507 (2013).

[6] M. T. Hansen and S. R. Sharpe, Phys. Rev. D 90, 116003 (2014).

[7] M. T. Hansen and S. R. Sharpe, Phys. Rev. D 92, 114509 (2015).

[8] M. T. Hansen and S. R. Sharpe, Phys. Rev. D 93, 014506 (2016).

[9] M. T. Hansen and S. R. Sharpe, Phys. Rev. D 93, 096006 (2016); 96, 039901(E) (2017).

[10] M. T. Hansen and S. R. Sharpe, Phys. Rev. D 95, 034501 (2017).

[11] R. A. Briceño, M. T. Hansen, and S. R. Sharpe, Phys. Rev. D 95, 074510 (2017).

[12] S. Kreuzer and H.-W. Hammer, Phys. Lett. B 694, 424 (2011).

[13] S. Kreuzer and H.-W. Hammer, Eur. Phys. J. A 43, 229 (2010).

[14] S. Kreuzer and H.-W. Hammer, Phys. Lett. B 673, 260 (2009).

[15] S. Kreuzer and H.-W. Grießhammer, Eur. Phys. J. A 48, 93 (2012).

[16] S. R. Sharpe, Phys. Rev. D 96, 054515 (2017).

[17] H.-W. Hammer, J. Y. Pang, and A. Rusetsky, J. High Energy Phys. 09 (2017) 109.

[18] H.-W. Hammer, J.-Y. Pang, and A. Rusetsky, J. High Energy Phys. 10 (2017) 115.

[19] Y. Meng, C. Liu, U.-G. Meißner, and A. Rusetsky, Phys. Rev. D 98, 014508 (2018).

[20] M. Döring, H.-W. Hammer, M. Mai, J.-Y. Pang, A. Rusetsky, and J. Wu, Phys. Rev. D 97, 114508 (2018).
[21] M. Mai and M. Döring, Eur. Phys. J. A 53, 240 (2017).

[22] R. A. Briceño, M. T. Hansen, and S. R. Sharpe, Phys. Rev. D 98, 014506 (2018).

[23] F. Romero-López, A. Rusetsky, and C. Urbach, Eur. Phys. J. C 78, 846 (2018).

[24] R. A. Briceño, M. T. Hansen, and S. R. Sharpe, Phys. Rev. D 99, 014516 (2019).

[25] M. Mai and M. Döring, Phys. Rev. Lett. 122, 062503 (2019).

[26] P. Guo and T. Morris, Phys. Rev. D 99, 014501 (2019).

[27] P. Guo, M. Döring, and A. P. Szczepaniak, Phys. Rev. D 98 , 094502 (2018).

[28] T. D. Blanton, F. Romero-López, and S. R. Sharpe, J. High Energy Phys. 03 (2019) 106.

[29] M. T. Hansen and S. R. Sharpe, arXiv:1901.00483.

[30] M. Lüscher, Commun. Math. Phys. 105, 153 (1986).

[31] K. Huang and C. N. Yang, Phys. Rev. 105, 767 (1957).

[32] T. T. Wu, Phys. Rev. 115, 1390 (1959).

[33] S. Tan, Phys. Rev. A 78, 013636 (2008).

[34] S. R. Beane, W. Detmold, and M. J. Savage, Phys. Rev. D 76, 074507 (2007).

[35] W. Detmold and M. J. Savage, Phys. Rev. D 77, 057502 (2008).

[36] E. Braaten and H.-W. Hammer, Phys. Rep. 428, 259 (2006).

[37] V. Bernard, M. Lage, U.-G. Meißner, and A. Rusetsky, J. High Energy Phys. 08 (2008) 024.

[38] M. Göckeler, R. Horsley, M. Lage, U.-G. Meißner, P. E. L. Rakow, A. Rusetsky, G. Schierholz, and J. M. Zanotti, Phys. Rev. D 86, 094513 (2012).

[39] F. Romero-López, A. Rusetsky, and C. Urbach, Phys. Rev. D 98, 014503 (2018).

[40] H. W. Hammer and T. Mehen, Phys. Lett. B 516, 353 (2001).

[41] C. Ji, D. R. Phillips, and L. Platter, Ann. Phys. (Amsterdam) 327, 1803 (2012). 